\title{
Interdecadal Variation of ENSO Predictability in Multiple Models
}

\author{
Youmin Tang, Ziwang Deng, Xiaobing Zhou, and Yanje Cheng \\ Environmental Science and Engineering, University of Northern British Columbia, Prince George, British Columbia, Canada \\ DAKE CHEN \\ Lamont-Doherty Earth Observatory, Columbia University, Palisades, New York, and State Key Laboratory of Satellite Ocean \\ Environment Dynamics, Hangzhou, China
}

(Manuscript received 16 August 2007, in final form 31 January 2008)

\begin{abstract}
In this study, El Niño-Southern Oscillation (ENSO) retrospective forecasts were performed for the 120 yr from 1881 to 2000 using three realistic models that assimilate the historic dataset of sea surface temperature (SST). By examining these retrospective forecasts and corresponding observations, as well as the oceanic analyses from which forecasts were initialized, several important issues related to ENSO predictability have been explored, including its interdecadal variability and the dominant factors that control the interdecadal variability.

The prediction skill of the three models showed a very consistent interdecadal variation, with high skill in the late nineteenth century and in the middle-late twentieth century, and low skill during the period from 1900 to 1960. The interdecadal variation in ENSO predictability is in good agreement with that in the signal of interannual variability and in the degree of asymmetry of ENSO system. A good relationship was also identified between the degree of asymmetry and the signal of interannual variability, and the former is highly related to the latter. Generally, the high predictability is attained when ENSO signal strength and the degree of asymmetry are enhanced, and vice versa. The atmospheric noise generally degrades overall prediction skill, especially for the skill of mean square error, but is able to favor some individual prediction cases. The possible reasons why these factors control ENSO predictability were also discussed.
\end{abstract}

\section{Introduction}

It has been of great interest to identify the sources and processes that limit the predictability of ENSO. Typically there are two hypotheses to explain the loss of predictability with forecast lead time. The first argues that the loss of predictability is due to the chaotic behavior of the nonlinear dynamics of the coupled system (e.g., Jin et al. 1994; Chen et al. 2004), whereas the second attributes it to the stochastic nature of the coupled system characterized by weather noise and other high-frequency variations, such as westerly wind bursts (WWB) and the Madden-Julian oscillation (MJO; e.g., Penland and Sardeshmukh 1995; Kleeman and Moore 1997; Vecchi and Harrison 2003; Moore et

Corresponding author address: Youmin Tang, Environmental Science and Engineering, University of Northern British Columbia, Prince George, BC V2N 4Z9, Canada.

E-mail: ytang@unbc.ca al. 2006; Gebbie et al. 2007). It is still not clear to date which regime plays the dominant role in controlling the variation of ENSO predictability.

A widely used strategy in studying ENSO predictability is to perform retrospective forecast experiments using dynamical models, by which variations in predictability and possible mechanisms responsible for the variations are investigated (e.g., Kirtman and Schopf 1998; Latif et al. 1998; Tang et al. 2005). Until recently, such retrospective forecasts only cover time periods of 20-40 yr because of the lack of long-term observations (such as wind stress). If ocean data assimilation is used for model initialization, this becomes even more serious a problem because of the still shorter ocean data record. Therefore, the period available for evaluating predictability contains relatively few ENSO cycles, basically precluding statistically robust conclusions. Chen et al. (2004) used the Lamont-Doherty Earth Observatory (LDEO) ENSO prediction model (referred to as the $\mathrm{Z}-\mathrm{C}$ model hereafter) to perform a retrospective 
forecast experiment of $148 \mathrm{yr}$ from 1856 to 2003. They found that ENSO predictability clearly has interdecadal variations. To date, this was the only work that studied ENSO predictability by extending realistic forecasts to a period of over $100 \mathrm{yr}$.

The prediction skill is usually model dependent. Thus, a challenging issue in ENSO predictability studies is to derive robust and general conclusions from long-term retrospective forecasts using multiple models. With two realistic ENSO prediction models and newly developed sea surface temperature (SST) assimilation approaches (Deng et al. 2008, hereafter DEN; Zhou et al. 2008, manuscript submitted to Ocean Modell., hereafter ZHO), we recently completed two longterm retrospective forecasts from 1881 to 2000. In this paper, we will examine ENSO predictability by these predictions. For comparison, the $\mathrm{Z}-\mathrm{C}$ model is used to generate another long-term retrospective forecast. The emphasis will be placed on the following two central questions: 1 ) are the variations in predictability shown in Chen et al. (2004) only the properties of the Z-C model or not, and 2) what causes the variations in ENSO predictability, namely, what limits ENSO predictability? These issues have not yet been well addressed.

This paper is structured as follows: Section 2 briefly describes the models, data, and initialization schemes. Section 3 examines the variation of prediction skill in three ENSO models for the period from 1881 to 2000. In section 4 , the possible mechanisms responsible for variations in ENSO predictability are discussed. A summary and discussion are given in section 5 .

\section{Models, data, initialization schemes, and metrics of prediction skill}

\section{a. Models}

Three coupled models are used in this study, including the Z-C model and two hybrid coupled modelsone is an oceanic general circulation model (OGCM) coupled to a linear statistical atmospheric model (HCM1), and the other is an intermediate complexity oceanic dynamical model coupled to a nonlinear statistical atmospheric model (HCM2). They are briefly described below.

\section{1) Z-C MODEL}

As the first dynamical coupled model used for ENSO prediction, the $\mathrm{Z}-\mathrm{C}$ model has been a benchmark in ENSO community for over two decades. It is composed of a Gill-type steady-state linear atmospheric model and a reduced-gravity oceanic model. The atmospheric circulation is forced by a heating anomaly that depends on the SST anomaly and moisture convergence, whereas the oceanic circulation is driven by surface wind. The SST is determined by the fully nonlinear thermodynamics in a surface mixed layer and by the linear dynamics through thermocline fluctuation.

The model version used in this study is LEDO5, identical to that in Chen et al. (2004). The model domain covers the tropical Pacific Ocean from $124^{\circ} \mathrm{E}-$ $80^{\circ} \mathrm{W}$ and $28.75^{\circ} \mathrm{S}-28.7^{\circ} \mathrm{N}$. The time step is 10 days.

\section{2) HCM1}

The ocean model is the latest version of Océan Parallélisé 9.0 (OPA9.0), an OGCM often used for oceanic modeling and prediction (e.g., Tang et al. 2004; Moore et al. 2006). Compared with the old version, the new development in OPA9.0 includes the partial cell bottom topography, advective bottom boundary layer parameterization, and double diffusion. Detailed formulation of the ocean model is described in Madec et al. (1998). The domain of the model used here is configured for the tropical Pacific Ocean from $30^{\circ} \mathrm{N}$ to $30^{\circ} \mathrm{S}$ and from $122^{\circ} \mathrm{E}$ to $70^{\circ} \mathrm{W}$, with $1.0^{\circ}$ horizontal resolution in the zonal direction and $0.5^{\circ}$ within $5^{\circ}$ of the equator, smoothly increasing to $2.0^{\circ}$ at $30^{\circ} \mathrm{N}$ and $30^{\circ} \mathrm{S}$ in the meridional direction. There are 31 vertical levels with 17 concentrated in the top $217 \mathrm{~m}$ of the ocean. The time step of integration is $1.5 \mathrm{~h}$ and all boundaries are closed, with no-slip conditions.

The statistical atmospheric model is a linear model that predicts the contemporaneous surface wind stress anomalies from SST anomalies (SSTA). It was constructed using observed SST and National Centers for Environmental Prediction-National Center for Atmospheric Research (NCEP-NCAR) reanalysis wind stress data from 1948 to 2002 (see below for data description) by the singular vector decomposition (SVD) method. The cross-validation scheme was used in the construction of the atmospheric model to ensure that the data used for validation was never used in training the model. In details, three atmosphere models, denoted as AM1, AM2, and AM3, were respectively constructed using the data of 1948-75, 1976-2002, and 1948-2002. The AM1 was used for the hindcast for 1976-2000, AM2 for 1948-75, and AM3 for 1881-1947. The first three SVD modes accounted for over $90 \%$ of total covariance and were used for constructing each statistical atmospheric model as in some similar work (e.g., Harrison et al. 2002; Galanti et al. 2003; Tang et al. 2004; Moore et al. 2006). Each atmospheric model was constructed for all calendar months.

During the initialization of the hybrid coupled model, the OGCM was forced by the sum of the associated wind anomalies computed from the atmospheric 
model and the observed monthly mean climatological wind stress. Full details of the model are given in DEN and Tang et al. (2004).

\section{3) $\mathrm{HCM} 2$}

HCM2 is composed of a nonlinear dynamical ocean model and a nonlinear empirical atmospheric model. The ocean model consists of a tropical Pacific model with six active layers allowing for an exchange of mass, momentum, and heat at each layer interface by a parameterization of entrainment. With a resolution of $1.5^{\circ} \times 1.5^{\circ}$, the model extends from $30^{\circ} \mathrm{N}$ to $30^{\circ} \mathrm{S}$ and from $123^{\circ} \mathrm{E}$ to $69^{\circ} \mathrm{W}$. The time step for integration is $2 \mathrm{~h}$.

The ocean model was first used to make a control run, with forcing by the Florida State University (FSU)observed wind stress (Goldenberg and O'Brien 1981) from 1961 to 1990 . The SST from the control run was then used as the predictor in the statistical models to reconstruct the atmospheric wind stress. After removing the climatological seasonal cycle, an empirical orthogonal function (EOF) analysis was performed separately on each anomaly dataset of SST and zonal and meridional wind stress to further extract the predictors and predictands. Only the first three EOF modes were used in the statistical model construction, as suggested by Latif et al. (1990) and Goswami and Shukla (1991). For the model SST, the first three EOF modes accounted for over $70 \%$ of the total variance, whereas the first three wind stress EOFs explained only $35 \%$ of the total variance, because of the presence of high-frequency oscillations and noise in the wind stress even though a three-point temporal running mean has been used before performing the EOF analysis. Finally, neural network (NN) models were applied to link these predictors and predictands to yield nonlinear atmospheric models. Similar to HCM1, the cross-validated scheme was used to construct the atmospheric model. HCM2 is identical to the hybrid coupled model used in Tang et al. (2001) and Tang and Hsieh (2003), and was used to issue real-time ENSO prediction in Experimental Long-Lead Forecast Bulletin (available online at http://grads.iges.org/ellfb/home.html). Further details of the ocean model and the construction of the nonlinear atmospheric model can be found in Tang et al. (2001) and Tang and Hsieh (2003).

\section{b. Data and the reconstruction of wind stress}

The data used in this study include the monthly global Extended Reconstructed SST (ERSST) dataset from 1854 to 2001 (online at http://www1.ncdc.noaa.gov/pub/ data/ersst), reconstructed by Smith and Reynolds
(2004) with $2^{\circ}$ latitude $\times 2^{\circ}$ longitude resolution. Because of the relatively poor quality of the dataset prior to 1878 , we focus on the period from 1881 to 2001 in this study. The data domain is configured for the tropical Pacific Ocean. This dataset is used for HCM1 and HCM2. For the Z-C model, the Kaplan extended SST dataset from 1856 to 2003 is used, with a spatial coverage of $5^{\circ}$ latitude $\times 5^{\circ}$ longitude (Chen et al. 2004).

The monthly NCEP-NCAR surface wind of the tropical Pacific Ocean from 1948 to 2001 was used to construct statistical atmospheric models and reconstruct the wind stress of the tropical Pacific Ocean from 1881 to 1947 . The NCEP-NCAR reanalysis dataset is interpolated onto a $2.5^{\circ} \times 2.5^{\circ}$ global grid. The monthly wind stress is obtained using a bulk aerodynamic formula (DEN).

To perform long-term hindcasts with HCMs, the past wind stress data are required to initialize forecasts. Empirical atmospheric models using SST to predict wind have been widely used in ENSO studies (e.g., Syu and Neelin 2000; Tang et al. 2001; Tang and Hsieh 2003). It has been found that such statistically estimated wind can produce better prediction skill than the observed wind when used to initialize forecasts (Syu and Neelin 2000; Tang and Hsieh 2002). Using SST as a predictor and SVD techniques, we reconstructed a long-term wind stress dataset from 1881 to 1947 . Forced by the reconstructed wind stress, the ocean models of both HCM1 and HCM2 generated a very good ENSO simulation for the period from 1881 to 2000 , with the correlation coefficient over 0.7 between the modeled and observed Niño-3.4 SSTA indices (averaged SSTA over the region of $5^{\circ} \mathrm{N}-5^{\circ} \mathrm{S}, 170^{\circ}-120^{\circ} \mathrm{W}$; see DEN; ZHO). It should be mentioned that wind stress is not required to initialize the Z-C model (Chen et al. 2004). The forecast of the $\mathrm{Z}-\mathrm{C}$ model is initialized by directly nudging SST into the coupled model.

\section{c. Data assimilation and the initialization of the forecast}

A very important task in ENSO predictions is to optimize the oceanic initial conditions. It has been found that the assimilation of subsurface in situ observations and satellite altimetry can significantly improve model skills (e.g., Tang and Hsieh 2003; Tang et al. 2004; Zheng et al. 2007). However, the oceanic subsurface observations and satellite altimetry are too short for this study; the only way is to assimilate SST to initialize forecasts. Usually SST is a prognostic variable in ocean models, and the general procedure of SST assimilation is to optimally insert it into the models (e.g., Rosati et al. 1997; Syu and Neelin 2000; Tang and Hsieh 2003). However, this strategy could lead to serious imbalances 
between the thermal and dynamical fields during the assimilation cycle (Tang and Kleeman 2002). Therefore, a key issue for SST assimilation is to alleviate the imbalances.

Three different assimilation algorithms were respectively applied to individual ocean models. For the Z-C model, we directly applied the nudging scheme used in Chen et al. (2004). With the aid of an interactive bias correction, the initialization scheme produced very good ENSO prediction skills (Chen et al. 2004). For HCM1, the ensemble Kalman filter (EnKF) was used to assimilate the SST anomaly into the OGCM, in which the background error covariance (BEC) matrix was constructed by ensemble members of different model levels to transfer the corrections in SST into the subsurface temperatures (DEN). The SST assimilation with the EnKF scheme leads to considerable improvement in modeling subsurface temperatures and ENSO forecasts. For HCM2, a specific approach to construct BEC was developed to transfer the information of SST into subsurface temperatures during the SST assimilation cycle. With the optimal interpolation (OI), the designed BEC produced ENSO prediction skills comparable with those in the $\mathrm{Z}-\mathrm{C}$ model $(\mathrm{ZHO})$. The details of the SST assimilation schemes and their performances in HCM1 and HCM2 were presented, respectively, in DEN and ZHO. We will use these assimilation schemes to initialize forecasts in this study. For convenience, we refer hereafter to the model SST from these assimilation schemes as analyzed SST or SST analysis. These analyzed SST fields will be used to study the possible factors that control the variation of predictability in the following sections.

\section{d. Metrics of prediction skill}

Typical metrics used to evaluate prediction skill includes the anomaly correlation coefficient $R$, mean square error (MSE), and some of their derivatives, as defined below. The seasonal cycle has always been removed from forecasts and observations prior to measuring prediction skills, unless otherwise indicated.

(i) The anomaly correlation skill $R$ and MSE are traditionally defined as

$$
R(t)=\frac{\sum_{i=1}^{N}\left[T_{i}^{p}(t)-\mu^{p}\right]\left[T_{i}^{o}(t)-\mu^{o}\right]}{\sqrt{\sum_{i=1}^{N}\left[T_{i}^{p}(t)-\mu^{p}\right]^{2}} \sqrt{\sum_{i=1}^{N}\left[T_{i}^{o}(t)-\mu^{o}\right]^{2}}},
$$

$$
\operatorname{MSE}(t)=\frac{1}{N-1} \sum_{i=1}^{N}\left[T_{i}^{p}(t)-T_{i}^{o}(t)\right]^{2},
$$

where $T$ is the index of Niño-3.4 SSTA, $p$ denotes forecasts, $o$ denotes observations, $t$ is the lead time of the forecast, $\mu^{p}$ is the mean of the forecasts, $\mu^{o}$ is the mean of observations, and $N$ is the number of samples used.

(ii) Mean square error of individual predictions (MSEIP) is used to measure the error of an individual forecast for all leads $L$ ( $L=12$ months), defined as

$$
\operatorname{MSEIP}=\frac{1}{L-1} \sum_{t=1}^{t=L}\left[T^{p}(t)-T^{o}(t)\right]^{2} .
$$

(iii) Correlation of individual forecasts (CIP) is an individual forecast correlated with its observed counterpart during the forecast period of $L$ months $(L=12)$, that is,

$$
\mathrm{CIP}=\frac{\sum_{t=1}^{L}\left(T_{t}^{p}-\mu^{p}\right)\left(T_{t}^{o}-\mu^{o}\right)}{\sqrt{\sum_{i=1}^{L}\left(T_{i}^{p}-\mu^{p}\right)^{2}} \sqrt{\sum_{i=1}^{L}\left(T_{t}^{o}-\mu^{o}\right)^{2}}} .
$$

\section{Variation of ENSO predictability}

Next we examine ENSO predictability by the three coupled models. A total of 480 forecasts, initialized from April 1881 to January 2001, were run starting at 3-month intervals (1 January, 1 April, 1 July, 1 October), and continued for 12 months for HCM1 and HCM2 and for 24 months for the Z-C model. The SST assimilation was performed to initialize the forecasts as introduced in section $2 \mathrm{c}$.

Figure 1 shows prediction skills $R$ (correlation) and MSE of the three models for the period from 1881 to 2000, where the predicted Niño-3.4 SSTA is compared against the observed value. Two kinds of forecasts from the $\mathrm{Z}-\mathrm{C}$ model are presented: direct forecasts from the $\mathrm{Z}-\mathrm{C}$ model (bold dashed line) and the corrected forecasts by simply adding a constant to forecasts at all leads $^{1}$ (bold line), which were used in Chen et al. (2004). Comparing these skills reveals that the corrected forecast of $\mathrm{Z}-\mathrm{C}$ is the best, and the forecast of HCM2 is comparable with, and even better than, the direct forecast of $\mathrm{Z}-\mathrm{C}$. The HCM1 is inferior to $\mathrm{Z}-\mathrm{C}$ and HCM2, but still shows a much better skill than

\footnotetext{
${ }^{1}$ The added constant is the difference between forecast and observation at the initial time. The reason for doing this is that the SST dataset used for initialization is detrended and thus different from that used for verification.
} 
(a)

(b)

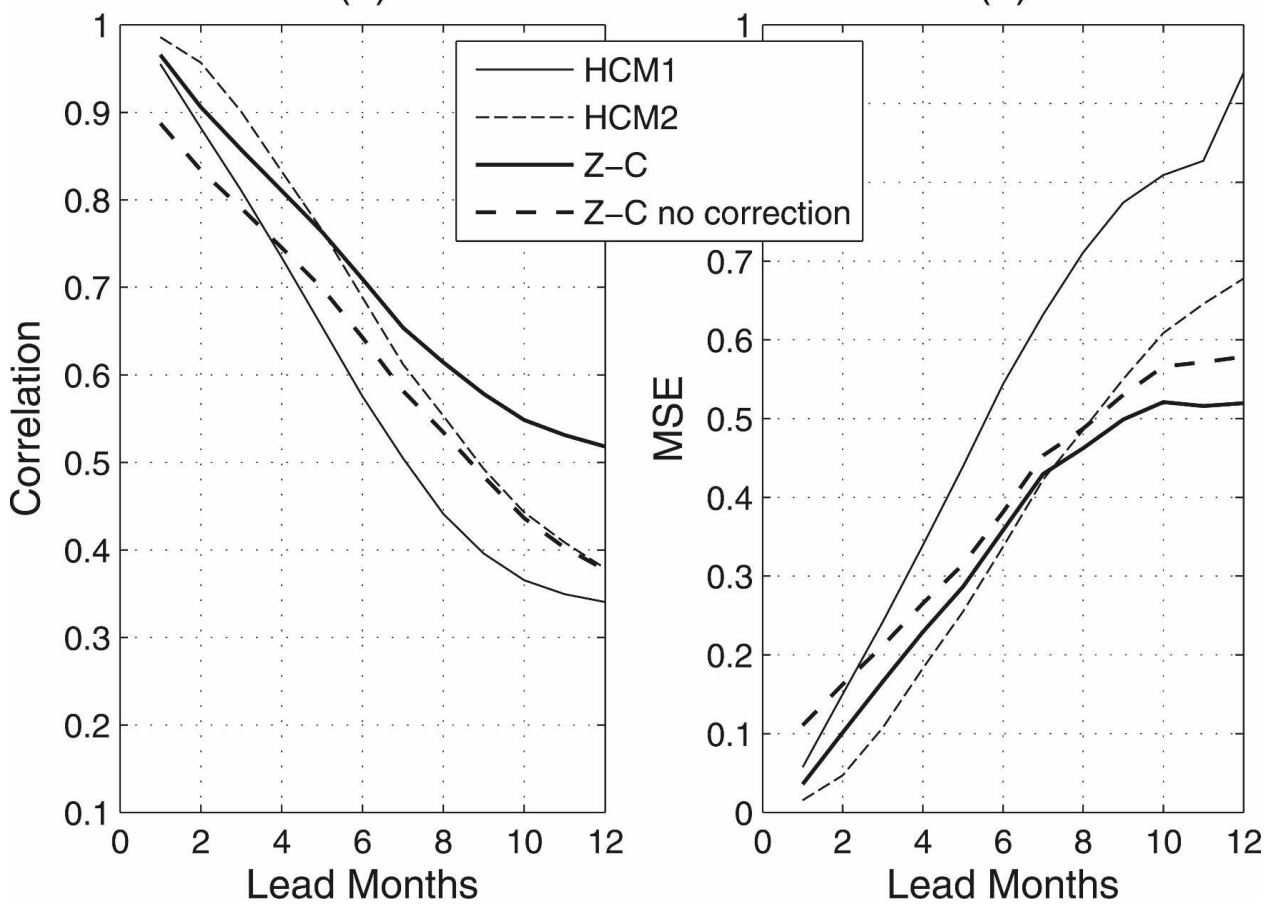

FIG. 1. Anomaly correlations $R$ and (b) MSE between the observed and the predicted values of the Niño-3.4 index, as a function of lead time, for the past $120 \mathrm{yr}$ from 1881-2000 for Z-C model, HCM1 and HCM2.

persistence (not shown). Shown in Fig. 2 is the predicted Niño-3.4 SSTA index at 6-month lead from 1881 to 2000 . As can be seen, the three models are able to predict most of the ENSO events, although they sometimes either produced false alarms or underpredicted the intensity, in particular for HCM1. It should be noted that the corrected forecast of the Z-C model was used in Fig. 2, and will always be used in following discussions unless otherwise indicated.

To examine the possible interdecadal variation of ENSO predictability, we calculated the prediction skills of six subperiods of $20 \mathrm{yr}$ each, including $R$ and MSE skill as shown in Figs. 3 and 4. It is evident in Fig. 3 that the correlation skills are significantly different among these periods for all three models. Comparing Figs. $3 \mathrm{a}-\mathrm{c}$ reveals a considerable consistency of variations in correlation skill among the three models. For example, high prediction skills always appear in the late nineteenth century and the middle-late twentieth century, that is, 1881-1900, 1961-80, and 1981-2000, whereas the periods of 1901-20, 1921-40, and 1941-60 have relatively low prediction skills. A similar consistency is also visible in the MSE skill as shown in Fig. 4, although it is not as obvious as in Fig. 3.

It is worth exploring the interdecadal variation of persistence skill for the period of 1881-2000. We re- peated the above analysis but used persistent prediction instead of model prediction, as shown in Fig. 5. Here the persistent prediction was obtained by persisting the observed Niño-3.4 SSTA at the initial time through the whole period of prediction. Figures 5a,c show $R$ and MSE skill of persistence prediction using ERSST dataset. As can be seen, the persistence skills have differences among different periods after 3-6-month leads. However, they are very different from the interdecadal variation of ENSO predictability shown in Figs. 3 and 4. For example, the period of the middle-late twentieth century that has high predictability produces very low persistence skill, whereas the period of 194160 that has the worst predictability corresponds with a high persistence period. We also used the Kaplan SST dataset to produce persistent prediction of the Niño-3.4 SSTA index, and obtained similar results as shown in Figs. 5b,d. Thus, the interdecadal variation in ENSO predictability in the three models is not very related to the interdecadal variation in persistent skill. This is probably because the SSTA persistence is dominated by the SSTA signal at the frequencies over the 4-yr period, whereas the interdecadal variation in actual predictability is strongly related to the strength of SSTA signal at the frequencies of $2-4-y r$ periods (Deng and Tang 2008). 

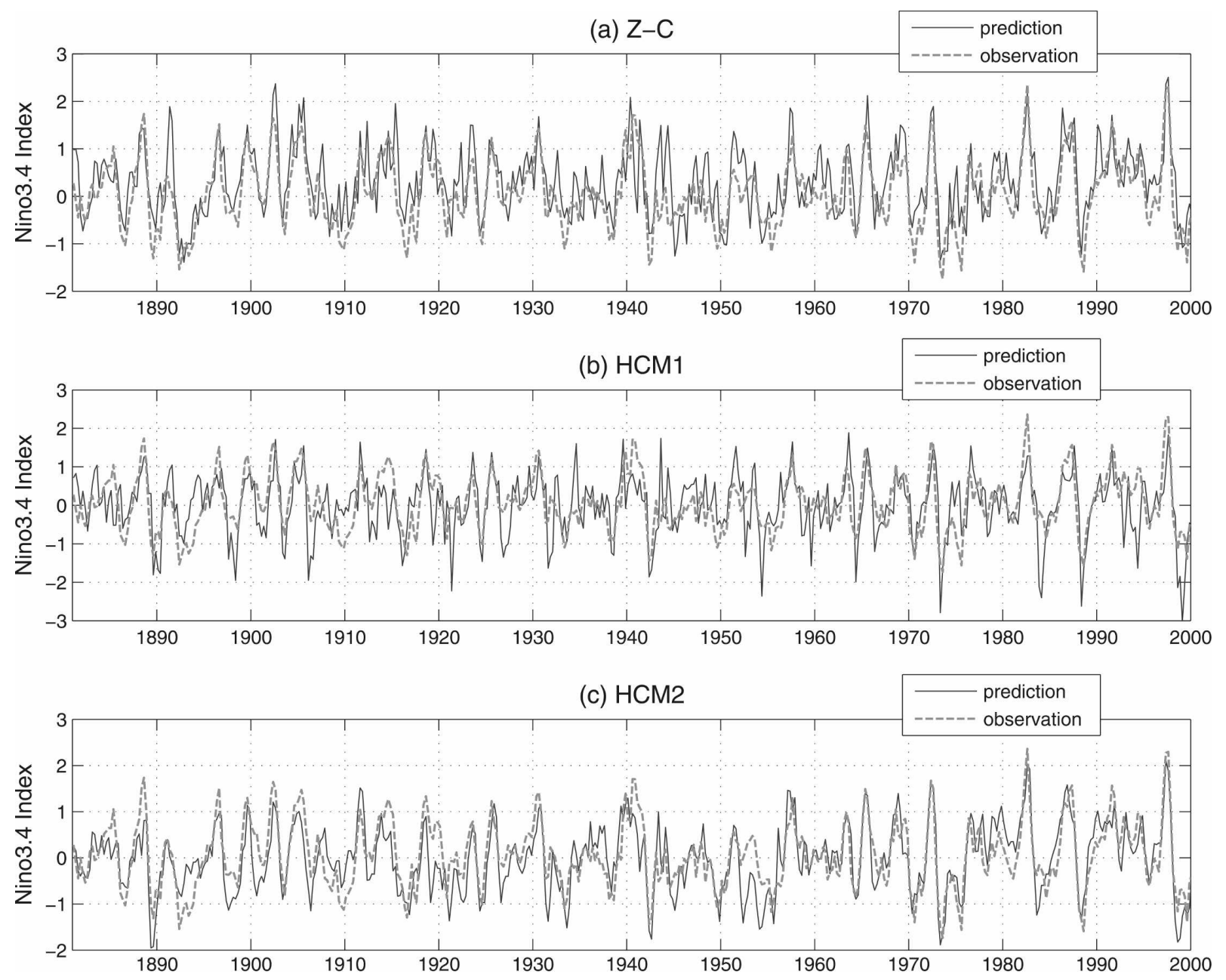

FIG. 2. The observed Niño-3.4 SSTA index and the predicted value of 6-month lead from (a) Z-C, (b) HCM1, and (c) HCM2.

The consistent temporal variations of prediction skills among the three models are further displayed in Fig. 6, which shows the averaged $R$ and MSE over 1-12month lead measured by a running window of $20 \mathrm{yr}$ from 1881 to 2000 (i.e., 1881-1900, 1882-1901, 1883$1902, \ldots, 1980-99,1981-2000) .^{2}$ As can be seen, the skill variations of the three models are in very good agreement, in particular for the correlation skill. Generally, good skills appear in the period around 18811900 and 1961-2000, whereas poor skills occur during the period from 1910 to 1960 . Such a variation feature is also manifested in Fig. 7, where different metrics are used to measure prediction skills.

\footnotetext{
${ }^{2}$ The overall skill measured during a $20-y r$ window is plotted at the middle point of the window in Fig. 6. For example, the skill at 1890 was calculated using the samples from 1881 to 1900 . The 20 -yr window is shifted by $1 \mathrm{yr}$ each time starting from 1881 until 2000.
}

In summary, there is a striking interdecadal variation of ENSO predictability in the past $120 \mathrm{yr}$ from 1881 to 2000 in the three prediction models. Generally, there is a high predictability in the late nineteenth century and in the middle-late twentieth century, and a low predictability from 1901 to 1960 . The interdecadal variation of ENSO predictability seems little dependent with models and metrics used in this study. All of the metrics and models used result in consistent conclusions.

One interesting question is whether or not the interdecadal variation in predictability reported above is mainly due to the differences of data and methods used in different decades. For example, one can speculate that the high prediction scores for the period from 1961 to 2000 are most probably due to better data quality because of the improvement of observation systems and the fact that models and wind reconstruction were trained using the data from part of this period. To explore this, we examined the simulation skill of the Niño- 
(a) ZC

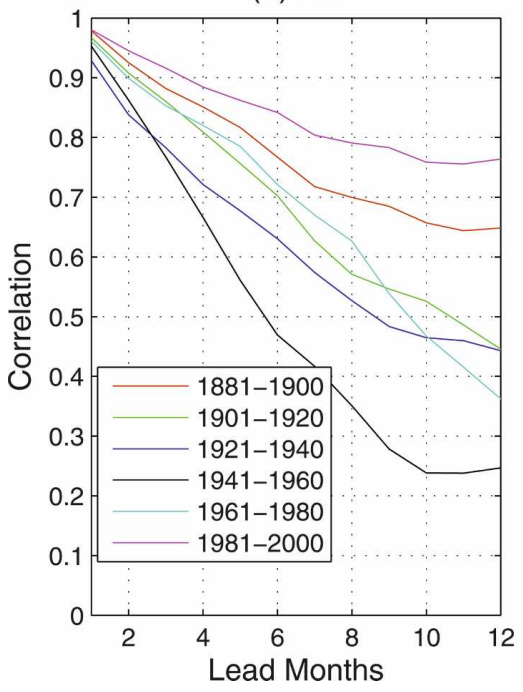

(b) HCM1

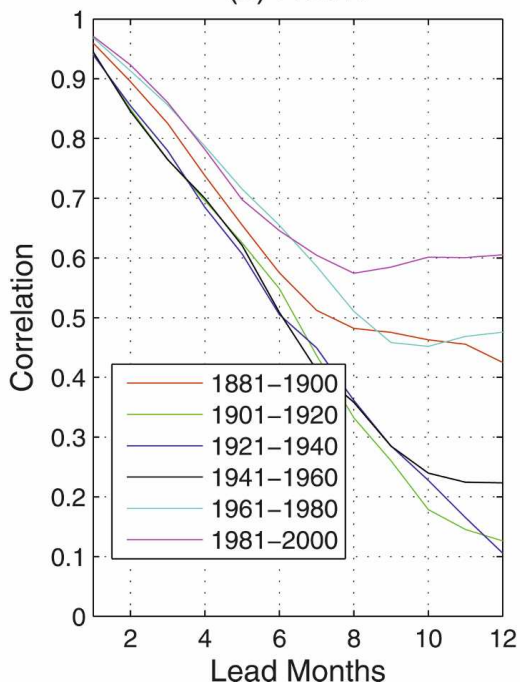

(c) $\mathrm{HCM} 2$

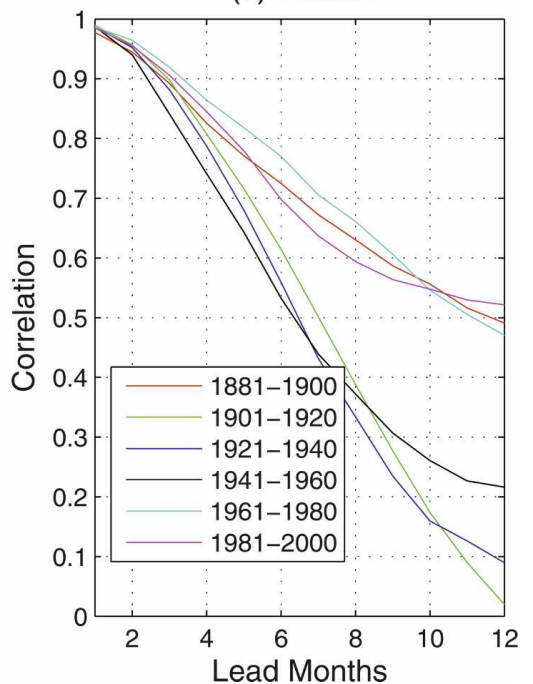

FIG. 3. Anomaly correlation $R$ between the observed and the predicted Niño-3.4 SSTA indices, as a function of lead time, for six consecutive 20-yr periods since 1881 for (a) the Z-C model, (b) HCM1, and (c) HCM2.

3.4 SSTA index forced by the reconstructed wind stress with the SST assimilation. The simulation skill indicates the quality of initial conditions of predictions and the model performance, both of which are inherent to the data quality and wind reconstruction. The impact of the differences of the data and methods used in different decades on the model prediction skill, if any existed, shall be most probably through initial conditions, such as initial SST. We verified the simulation skill using the Kaplan SST dataset for HCM1 and HCM2, and the ERSST dataset for the $\mathrm{Z}-\mathrm{C}$ model, to ensure that the simulation skill was obtained in a frame of a cross- validation scheme. Shown in Fig. 8 is the simulation skill computed in each running window of $20 \mathrm{yr}$ from 1881 to 2000 for the three models. As can be seen, the interdecadal difference of the simulation skill is not large in each model. The magnitude of variation is around 0.1 from the maximum to minimum during 1881-2000 for both $R$ and MSE. A comparison between Fig. 8 with Figs. 6 and 7 reveals that the interdecadal variation in predictability does not agree with that in the simulation skill. In contrast, their relationship seems out of phase in some decades. Thus, interdecadal variation in predictability is not due to model perfor- (a) ZC

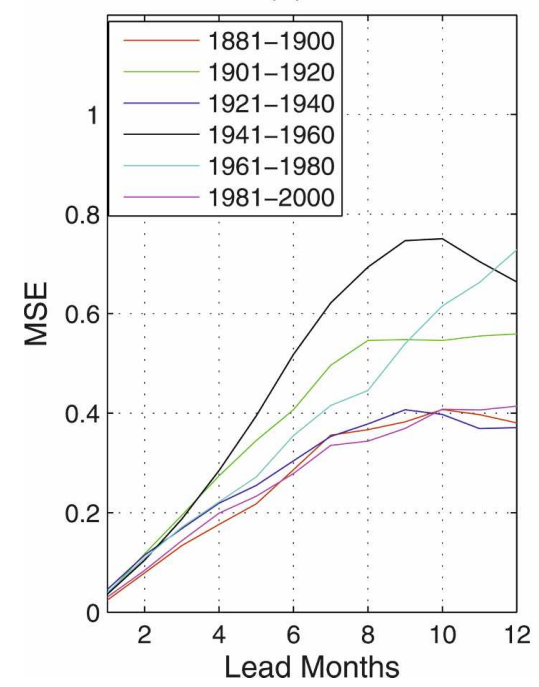

(b) HCM1

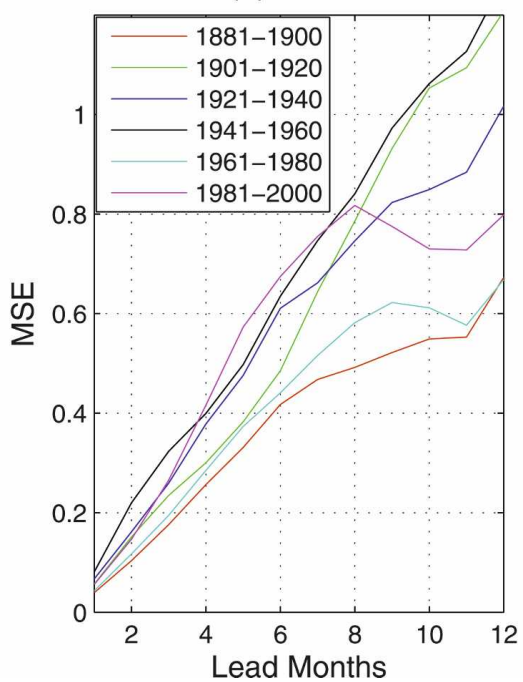

FIG. 4. Same as Fig. 3, but for MSE. (c) $\mathrm{HCM} 2$

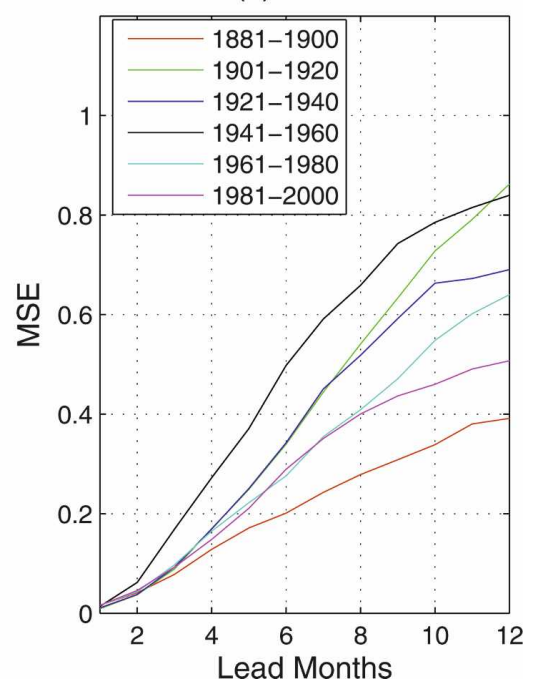


(a) ERSST
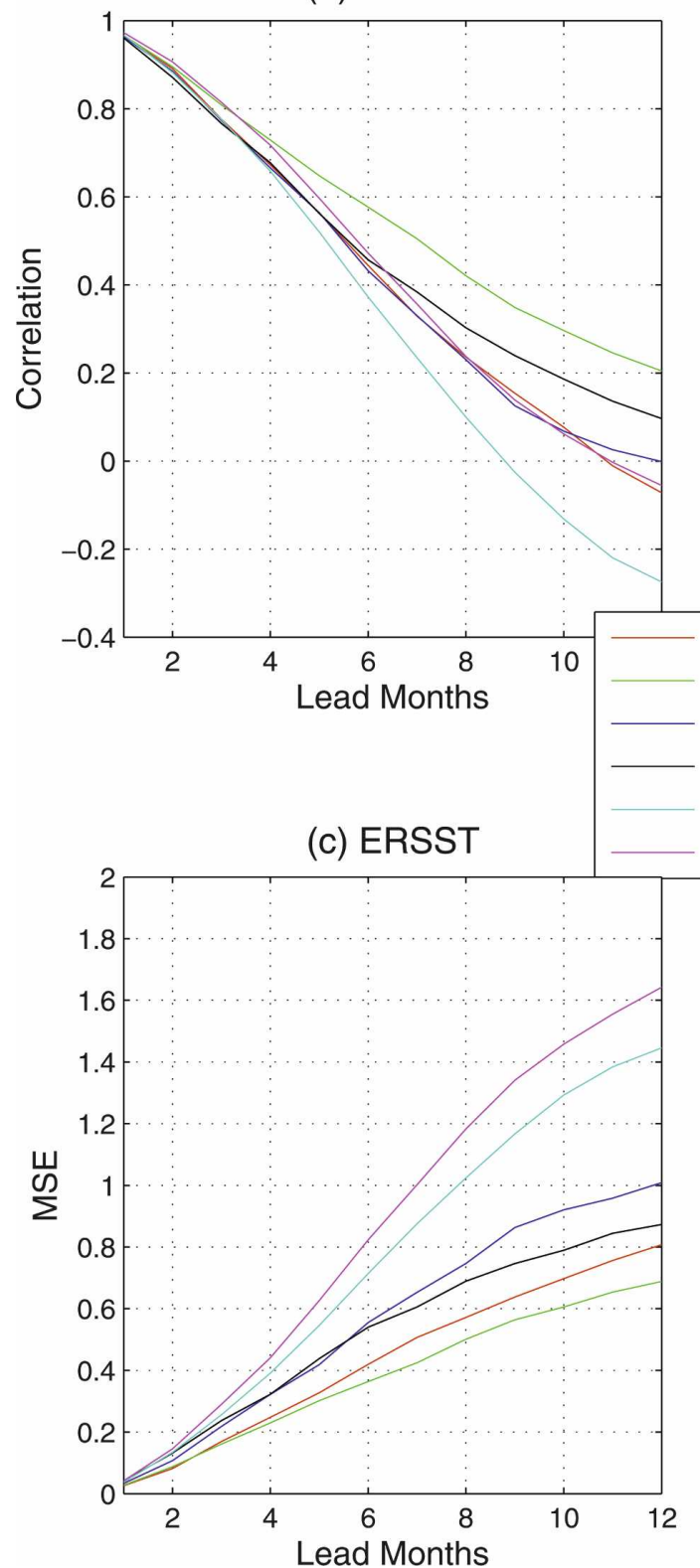

(b) Kaplan SST

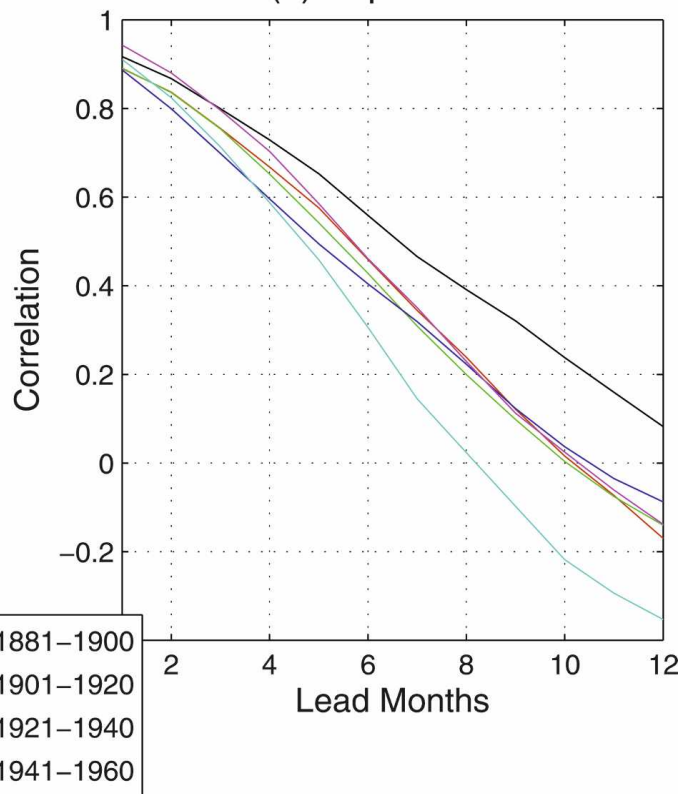

(d) Kaplan SST

FIG. 5. Persistence skill ( $R$ and MSE) of Niño-3.4 SSTA index, as a function of lead time, for six consecutive 20-yr periods since 1881. The persistence skill was obtained using the (a), (b) ERSST dataset and (c), (d) Kaplan SST.

mance associated with data quality and the wind stress reconstruction. This is further suggested by the fact that the high prediction skill also occurred during the period from 1881 to 1900. In next section, we will explore the possible reasons and sources of the interdecadal variation in predictability in detail.

\section{Sources to control ENSO predictability}

It is of interest to explore possible sources and mechanisms responsible for the interdecadal variation in ENSO predictability found in the preceding section. In this section, we thus turn to analyze the model forcing and initial conditions that determine prediction skills. It has been well recognized that the signal components present in initial fields play a critical role in determining ENSO prediction skills (e.g., Peng and $\mathrm{Ku}-$ mar 2005; Tang et al. 2005, 2008; Moore et al. 2006). Also, as mentioned in the introduction, nonlinearity and stochastic noise are thought to be two main sources to limit ENSO predictability. Therefore, we will examine the possible variations in the strength of the signal, 
(a)

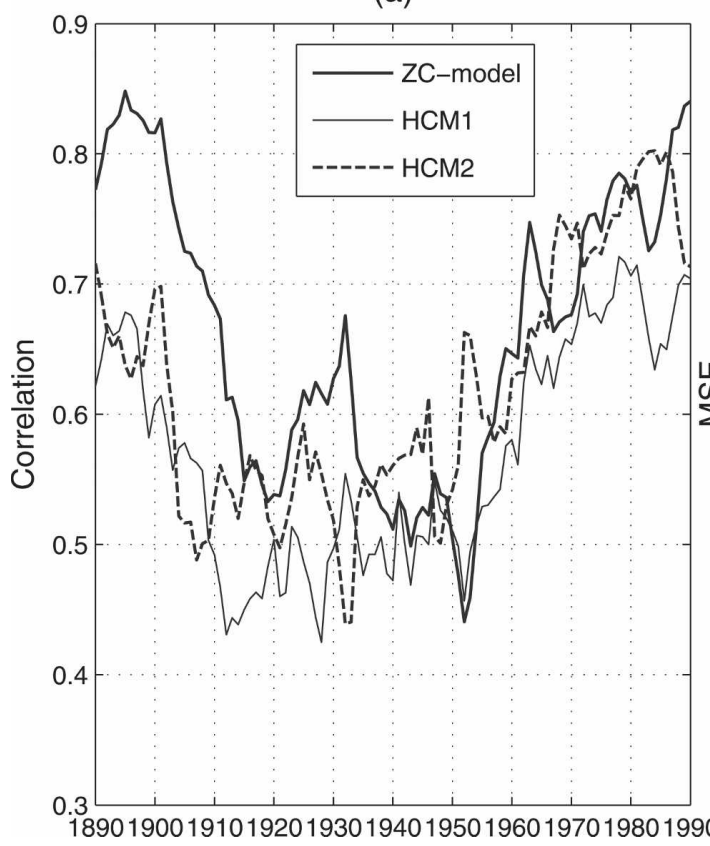

(b)

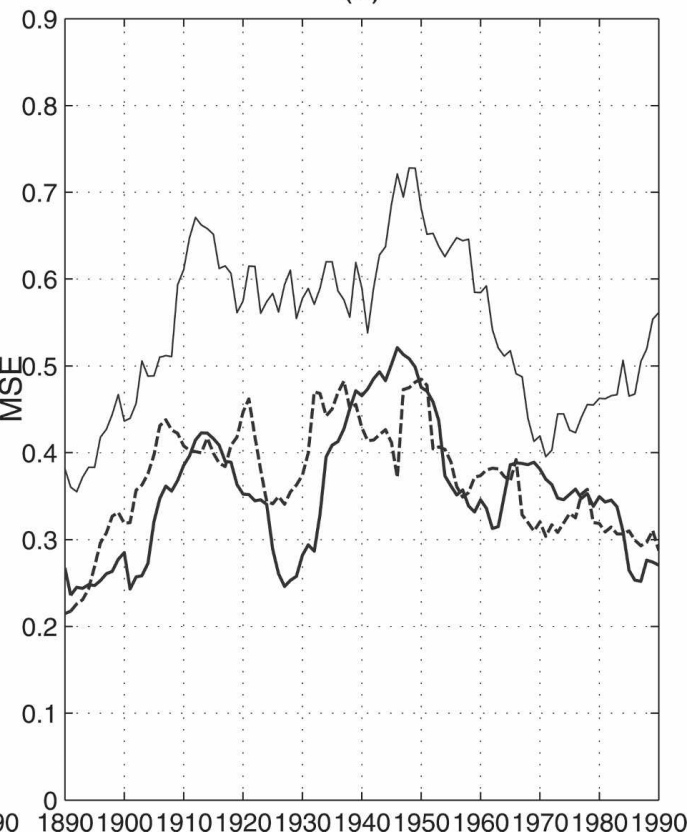

FIG. 6. (a) The average of anomaly correlation $R$ between the observed and the predicted Niño-3.4 SSTA indices over lead times of 1-12 months for three models: Z-C (bold solid line), HCM1 (thin-solid line), HCM2 (bolddashed line). The correlation $R$ was computed at each running window of 20-yr period from 1881 until 2000 (see context). (b) Same as (a), but for MSE.

nonlinearity, and noise using SST analysis from which forecasts were initialized. The emphasis will be placed on identifying possible relationships between these variables and ENSO predictability. To avoid complex-

(a)

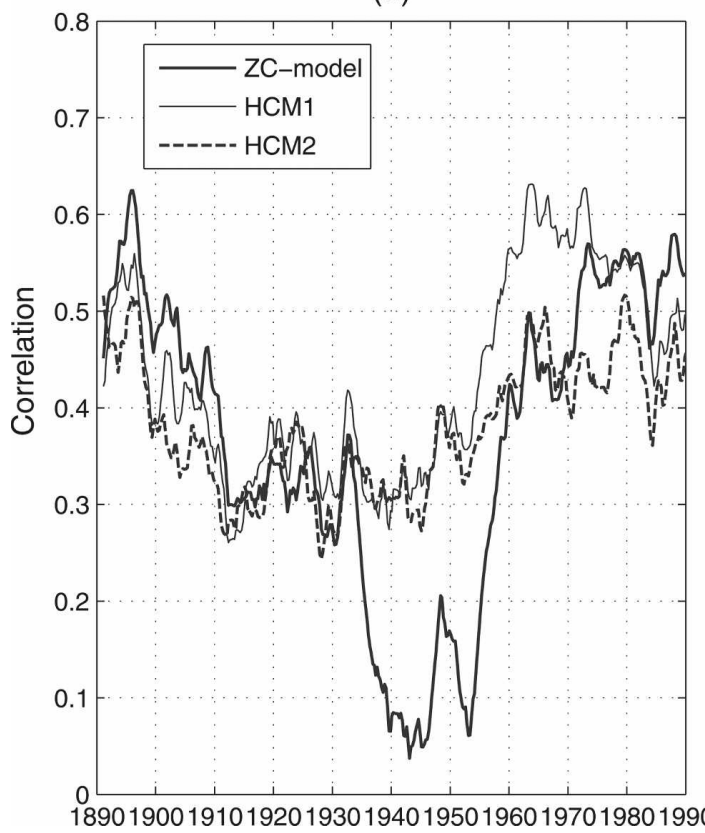

ity, we confine our investigation to the SST field, because the SST constitutes the most important signal in the ENSO system, and is used to initialize forecasts for the three models. Because the analyzed SST agrees

(b)

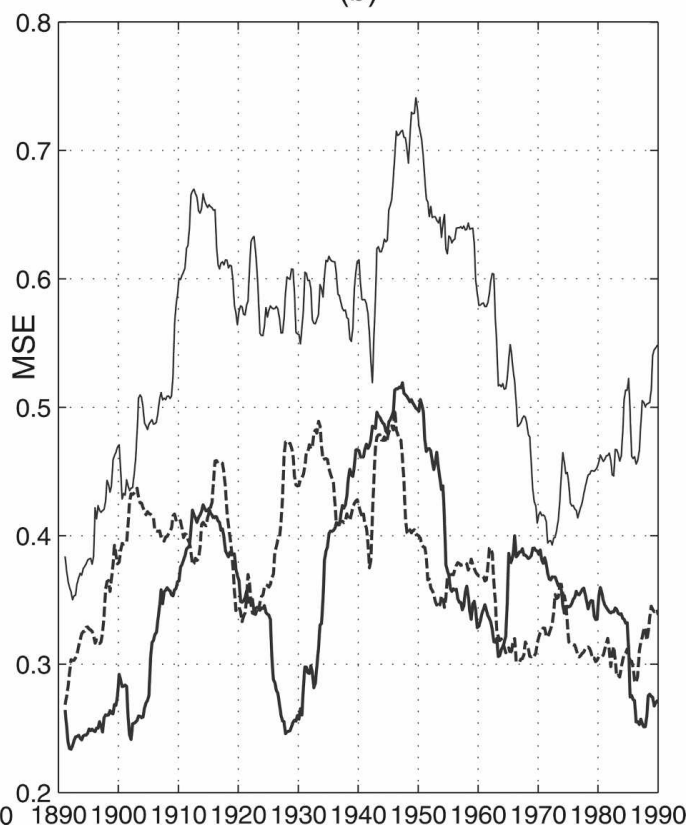

FIG. 7. The 20-yr running mean of (a) CIP and (b) MSEIP. Z-C (bold-solid line), HCM1 (thin solid line), HCM2 (bold dashed line). 
(a)

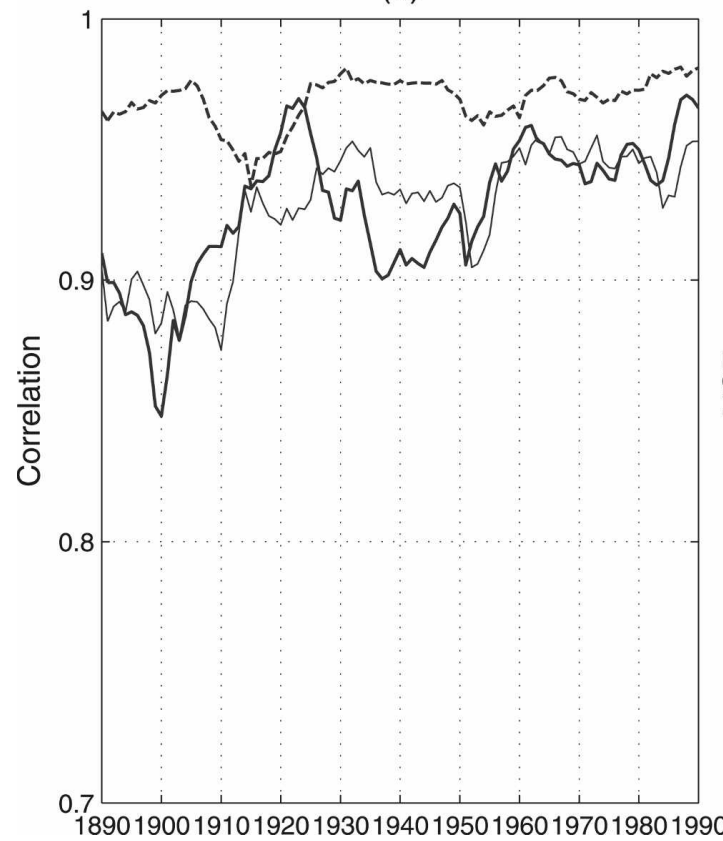

(b)

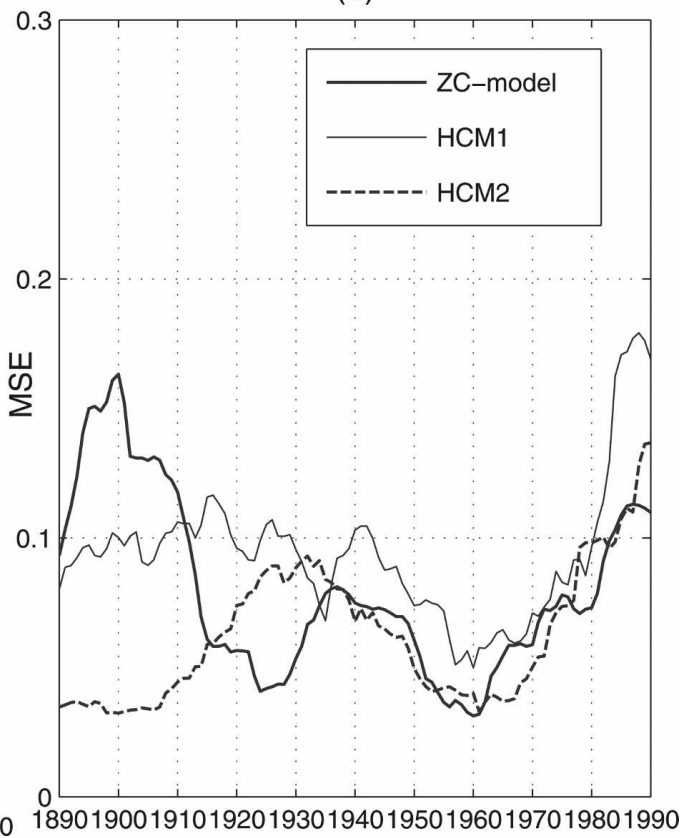

FIG. 8. (a) The anomaly correlation $R$ between the observed and simulated Niño-3.4 SSTA indices forced by reconstructed wind stress for three models: Z-C (bold solid line), HCM1 (thin solid line), and HCM2 (bold dashed line). The correlation $R$ was computed at each running window of 20-yr period from 1881 until 2000. (b) Same as (a), but for MSE.

with the observed counterpart very well, the following analyses explore the impact of anomalous features of the realistic ENSO system on ENSO predictability. In fact, the results from the analyzed SST are very similar to those from the observed SST.

\section{a. Variance and signal strength}

It has been shown in the literature that the signal strength present in initial conditions often dominate model prediction skills (e.g., Kleeman and Moore 1997; Tang et al. 2005, 2008). Several methods were applied to extract the ENSO signal in this study. The first method was to use the variance of the analyzed Niño3.4 SSTA index, computed for each running window of $20 \mathrm{yr}$ from 1881 to 2000 . The second method was to perform EOF for each running window of $20 \mathrm{yr}$, and the variance explained by the first mode was used to represent the intensity of the ENSO signal. The third method was to perform spectrum analysis for the analyzed Niño-3.4 SSTA index for each 20-yr running window, using the total spectrum power at the frequencies of 2-5 $\mathrm{yr}$ to represent the strength of ENSO signal. Figure 9 shows variations in prediction skills and in signals, demonstrating a significant relationship between the predictability and the signals in the three models. As can be seen, all of the methods of extracting
ENSO signals produced similar results. Like the interdecadal variability in prediction skills, ENSO signals also display an interdecadal variation. In the late nineteenth century, the signals were strong, and all models showed a large correlation $R$ and a low MSE. Since then the signal strength weakened and the skill continuously declined with time, both reaching a minimum around the 1940s-50s, beyond which both rebounded and increased with time until the 1960s. All models have a relatively good prediction skill from the 1960s, especially in the late twentieth century. Correspondingly, ENSO signals are also the strongest in these periods. Such a good relationship between ENSO signals and prediction skills holds not only for correlation $R$ but also for MSE skill. This relationship also holds for all three models used here.

It is of interest to explore the underlying physical interpretation of the relationship between signal and prediction skill. As found in Kleeman (2002) and Tang et al. (2005), the extra information that is provided by a forecast, called prediction utility, is highly associated with the signals present in the initial conditions. Because signals are large, more information will be produced compared with the climatological forecast, leading to a forecast that is likely to be reliable. Kleeman and Moore (1997) argued that the periods during which the slowly decaying eigenmodes are present with strong 


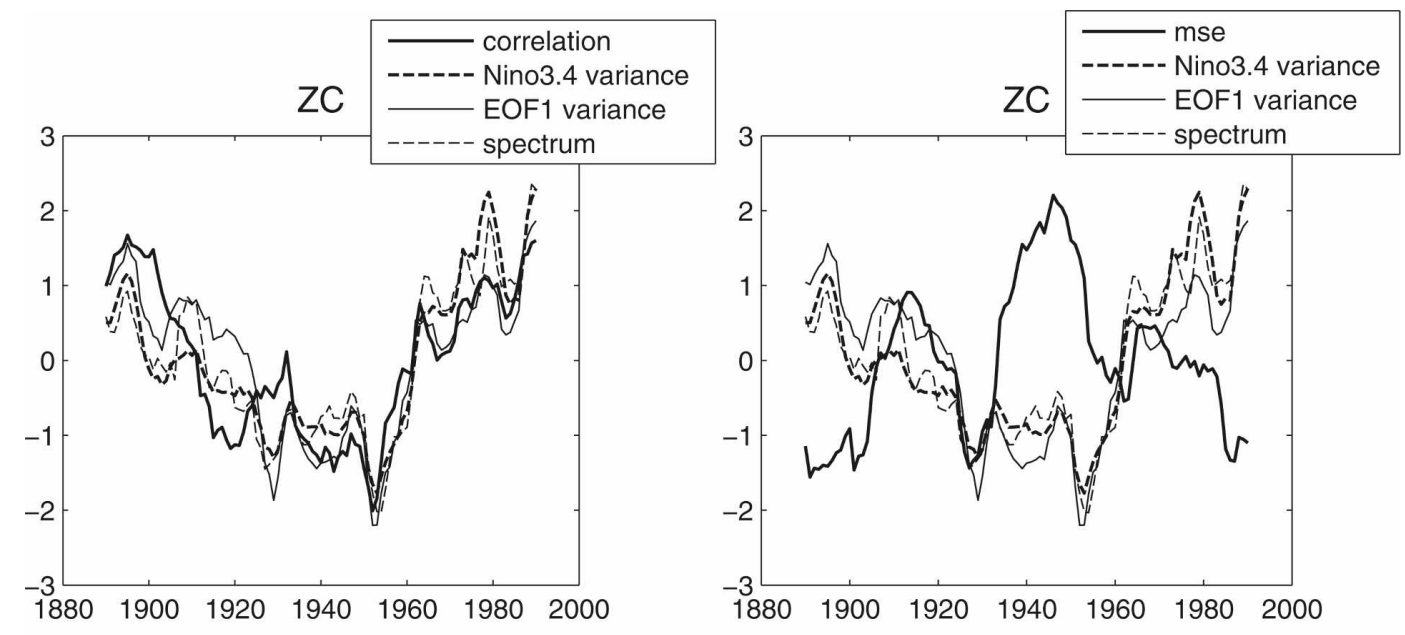

HCM1

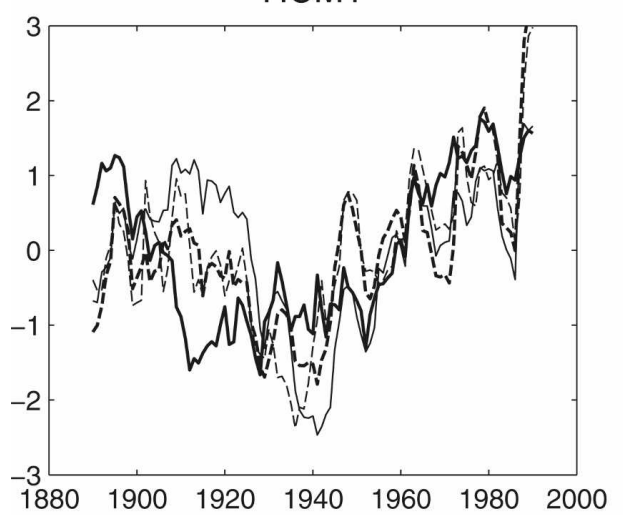

$\mathrm{HCM} 2$

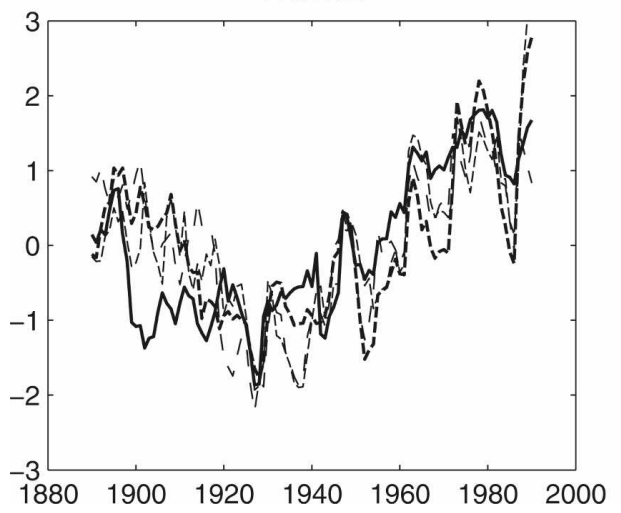

HCM1

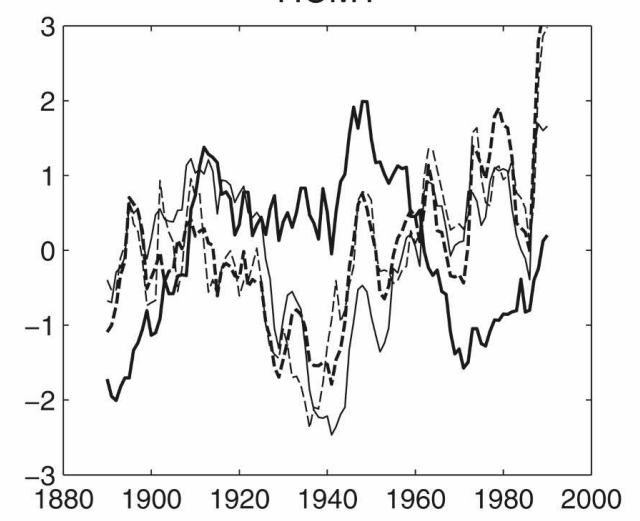

$\mathrm{HCM} 2$

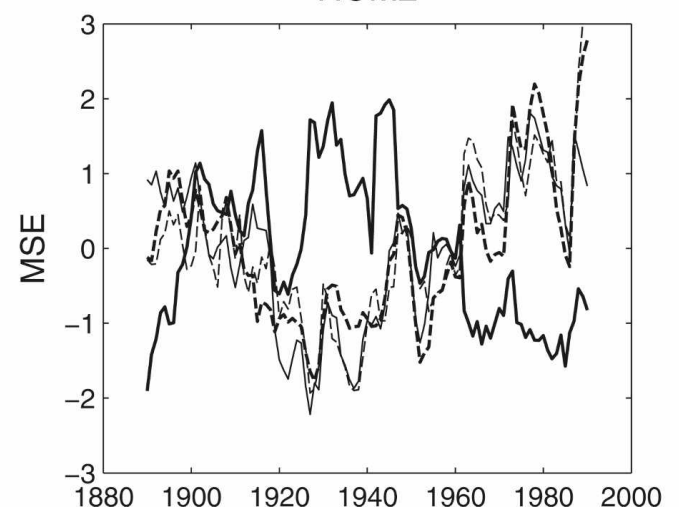

FIG. 9. The averaged prediction skill $R$ and MSE over 1-12-month leads of Niño-3.4 SSTA index and the signals estimated by several methods (see context), for Z-C, HCM1, and HCM2. The normalization was applied prior to plotting.

signals are periods that should be intrinsically more predictable because such modes are able to "resist" dissipation by more chaotic or stochastic components of the system.
Figure 10 is a scatterplot of prediction skills against variances of the analyzed Niño-3.4 SSTA index, both computed in each running window of $20 \mathrm{yr}$ from 1881 to 2000, for the three models. A good linear relationship 
(a) ZC

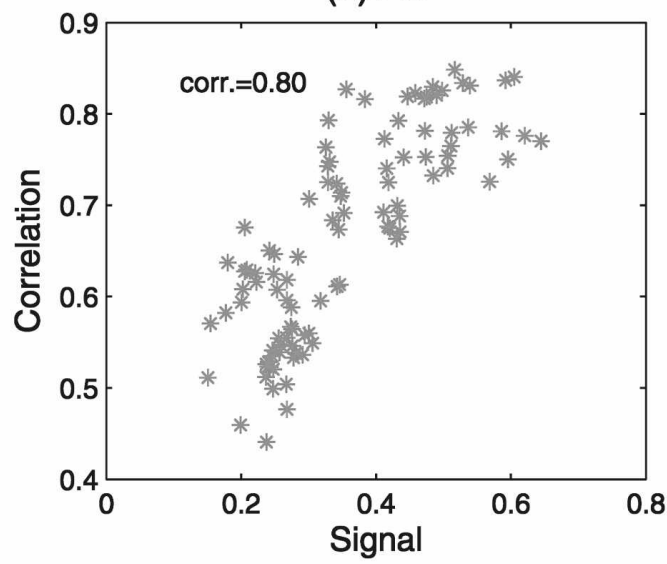

(c) HCM1

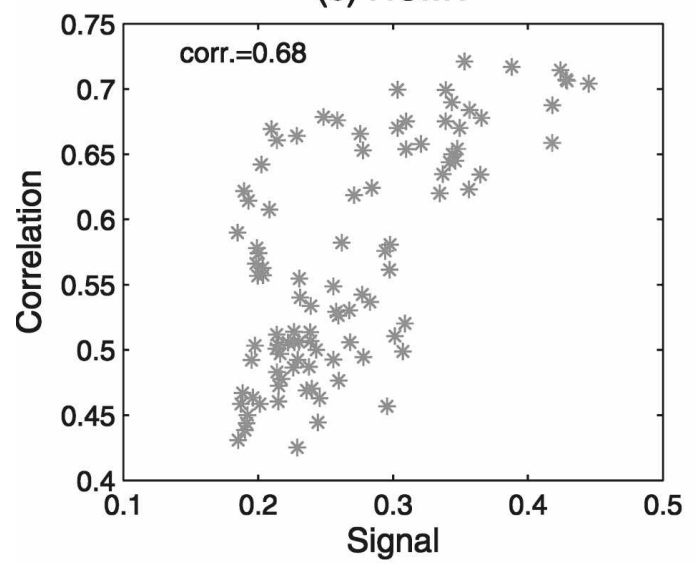

(e) HCM2

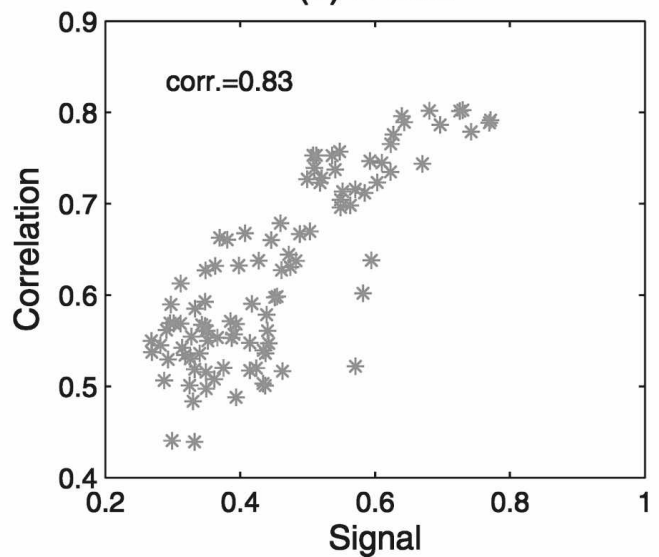

(b) ZC

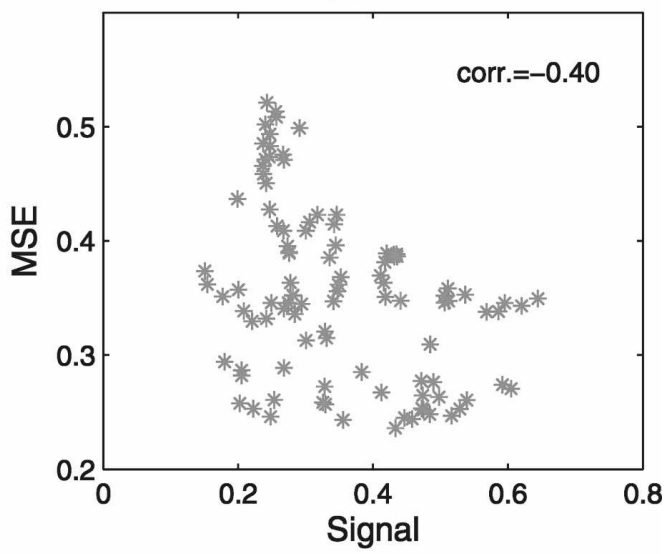

(d) HCM1

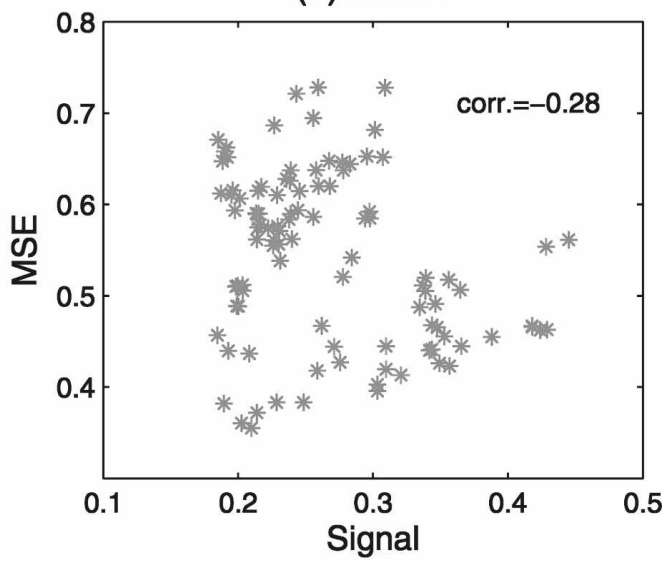

(f) $\mathrm{HCM} 2$

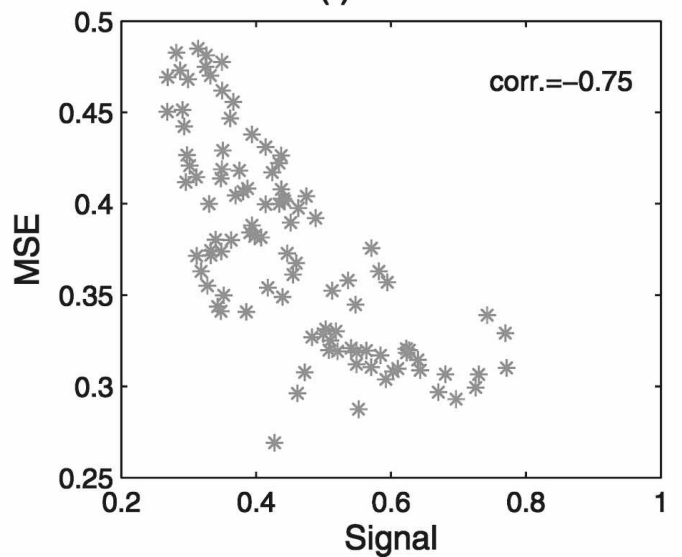

FIG. 10. Scatterplot of averaged prediction skill $R$ and MSE over 1-12-month leads against the variance of analyzed Niño-3.4 index, both calculated in each running window of $20 \mathrm{yr}$ from 1881 to 2000 .

can be found between the correlation skill and the variance for all models, especially for Z-C and HCM2. Also there is a good inverse relationship between MSE and the variance for $\mathrm{Z}-\mathrm{C}$ and $\mathrm{HCM} 2$. The correlation coefficients between the variances and prediction skills are shown in the upper corner of each panel. Compared with other two models, HCM1 shows the weakest relationship between the signals and prediction skills, 
which is, however, still statistically significant at the confidence level of $95 \%$. The relatively weak relationship in HCM1 is probably due to the fact that this model has a damped oscillation (DEN), leading to the signals present in initial conditions being quickly dissipated. $^{3}$

\section{b. Skewness and the degree of nonlinearity}

One basic characteristics of ENSO is the asymmetry between El Niño and La Niña (e.g., Wu and Hsieh 2003; Horling et al. 1997; An and Jin 2004; An 2004). It is most probably related to the nonlinear process of ENSO because the linear system could not induce the asymmetric feature. There is evidence in the observation and modeling that the asymmetry of ENSO is closely linked to the nonlinear process. For example, Jin et al. (2003) and An and Jin 2004 point out that the nonlinear dynamical heating could play important roles in the asymmetry between amplitudes of El Niño and La Niña events. In recent work, Perez et al. (2005) and Gebbie et al. (2007) argued that the asymmetry of atmospheric forcing causes the asymmetry of ENSO, through nonlinear feedback of the atmosphere and ocean.

In this section, we will investigate the possible impact of the cold-warm asymmetry on ENSO predictability. Statistically, the degree of asymmetry can be well characterized by the skewness, which has been widely applied to explore the nonlinear and asymmetric features of climate fields (e.g., Monahan and Dai 2004; An 2004). Thus, the skewness is used here to measure the degree of the nonlinearity of the ENSO system characterized by the asymmetry. It should be noticed that a non-Gaussian linear process could have nonzero skewness; thus, the following discussions are limited to the Gaussian process. ENSO could be thought of as an approximate Gaussian process, and the Gaussian assumption has been used and validated in many ENSO models (e.g., Penland and Sardeshmukh 1995; Kleeman 2002; Tang et al. 2008).

The skewness was calculated using the analyzed Niño-3.4 SSTA index in each window of $20 \mathrm{yr}$ running from 1881 to 2000 . The absolute skewness was used here to characterize the degree of asymmetry. For comparison, the absolute skewness and the prediction skill were both normalized as displayed in Fig. 11. As can be seen, the skewness obviously has an interdecadal variation that is in good agreement with the prediction skill.

\footnotetext{
${ }^{3}$ In a standard coupling run, the model Niño-3.4 SSTA index is damped to around $60 \%$ of the initial amplitude after 3-4 months, and then oscillates stably.
}

The periods that show good prediction skills, such as the late nineteenth century and the middle-late twentieth century, also have large absolute skewness. This holds for all three models, and for both correlation and MSE skills. The good relationship between the skewness and prediction skill is further shown in Fig. 12 for the three models. The value shown in the upper corner of each panel in Fig. 12 is the correlation coefficient between the skewness and the prediction skill, all of which are statistically significant at the confidence level of $99 \%$ with a two-tailed student's $t$ test.

The above finding suggests that the nonlinearity of the ENSO system leads to good predictability. This is mainly due to the nonlinearity characterized by skewness, which is highly related to the signal of ENSO variability. Shown in Fig. 13 is a comparison between the skewness and ENSO signal during the period from 1881 to 2000 for the three models, where the skewness was obtained as in Fig. 11 and ENSO signals were obtained as in Fig. 9. For comparison, normalization was applied to each variable here. As can be seen, the skewness is in very good agreement with the ENSO signal. The correlation coefficients between the skewness and signal in the three models are statistically significant at the $99 \%$ confidence level, with the averaged values of 0.76, 0.50, and 0.68 for Z-C, HCM1, and HCM2, respectively. This suggests that the interdecadal variation of skewness is most likely due to the interdecadal variation of ENSO strength. It is not surprising because strong ENSO events usually generate large asymmetric anomalies of SST, leading to large skewness. By analyzing observed SST data of 148 yr from 1856 to 2003, An (2004) also found that the variation in skewness of the tropical Pacific SST is highly related to the variation of SST amplitude. Thus, the positive relationship between the degree of nonlinearity and the predictability reflects the impact of the ENSO signal present in initial conditions on the prediction skill as discussed above. On the other hand, enhanced nonlinearity may be able to intensify the ENSO amplitude, meaning a large signal that is easy to predict as discussed above. Using the three-component Lorenz model and the Z-C model, Ye and Hsieh (2008, manuscript submitted to Nonlinear Processes Geophys.) also found that better predictability is attained when the nonlinearity of the models are adequately enhanced.

Nonlinear effects on the ENSO period have been widely discussed (e.g., Chang et al. 1996; Tang 2002; Eccles and Tziperman 2004). It should be noted that all models used here are intermediate or hybrid coupled models, and thus lack other realistic nonlinearities that have to be characterized by fully coupled general 
ZC

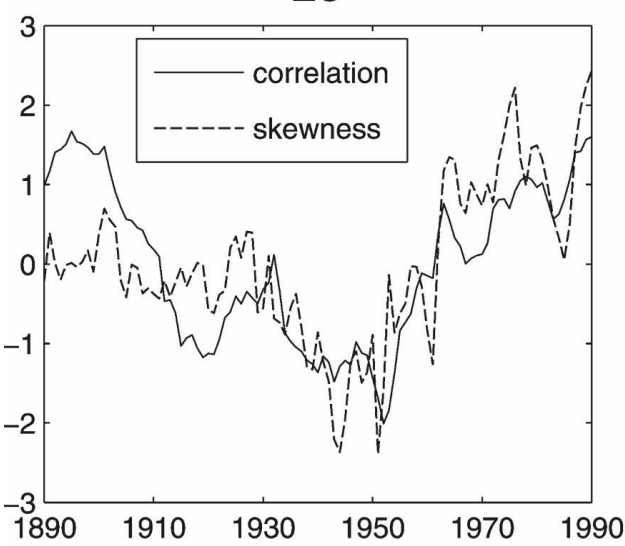

HCM1

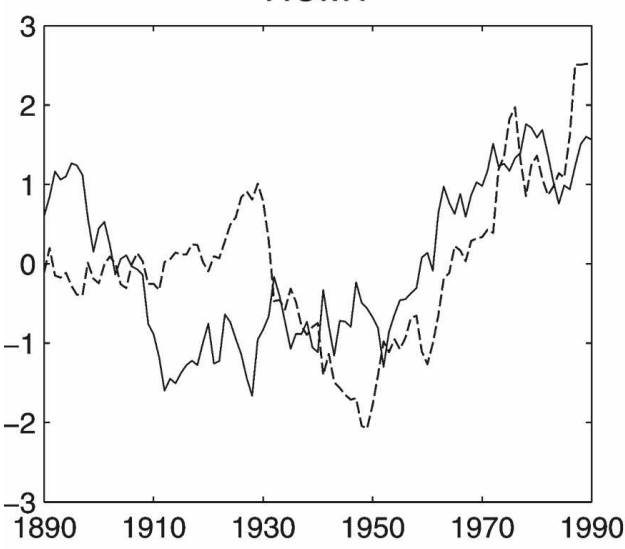

$\mathrm{HCM} 2$

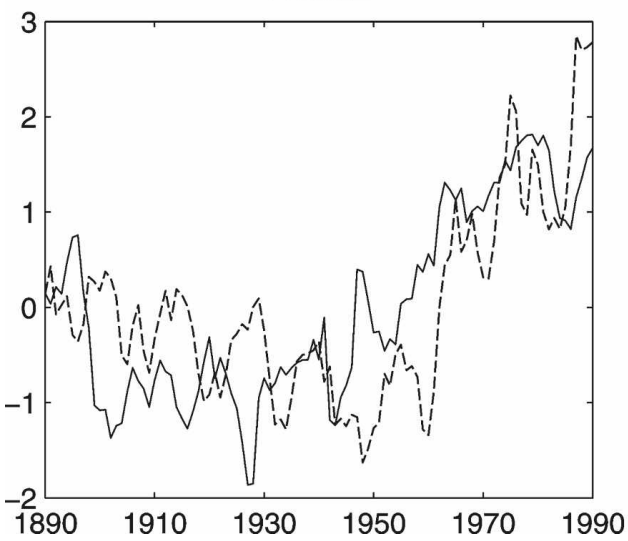

ZC

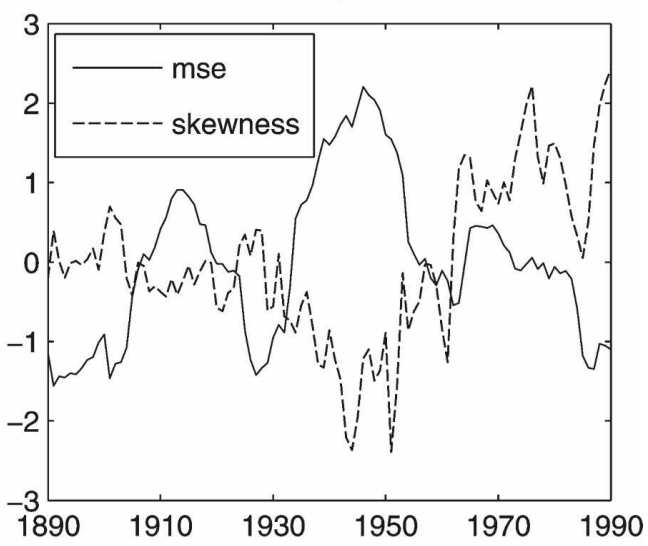

HCM1

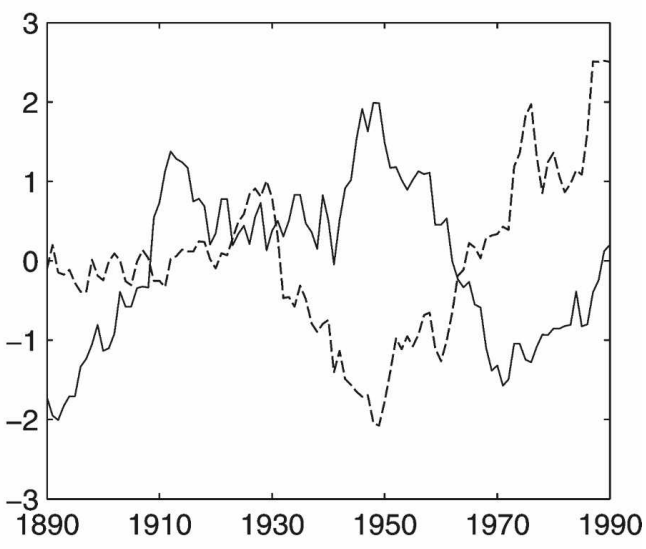

FIG. 11. The averaged prediction skill $R$ and MSE over lead times of 1-12 months and the skewness of the model Niño-3.4 SSTA index, both computed in each running window of $20 \mathrm{yr}$ from 1881 to 2000, for Z-C, HCM1, and HCM2. The normalization was applied prior to plotting for removing the unit. 
(a) ZC

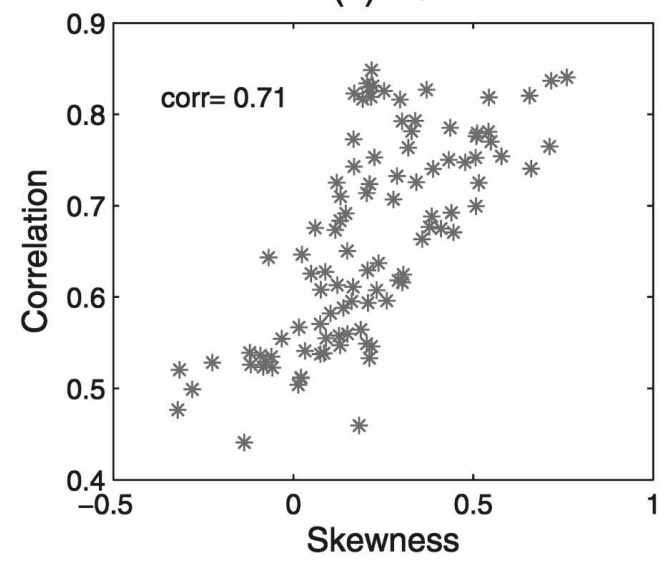

(c) HCM1

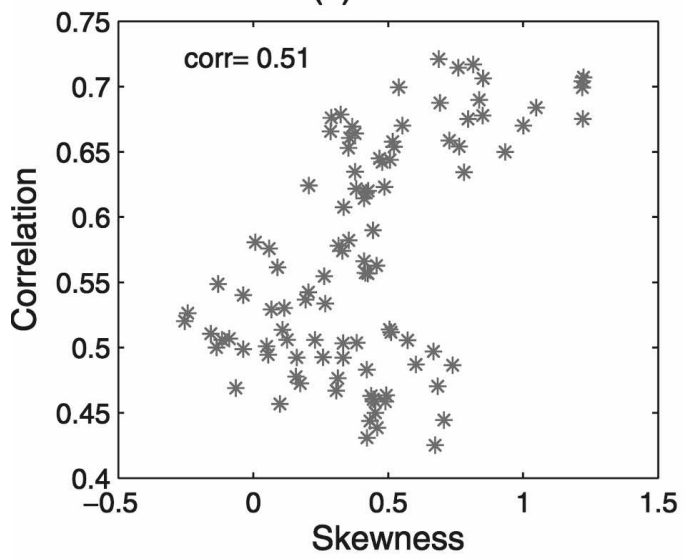

(e) HCM2

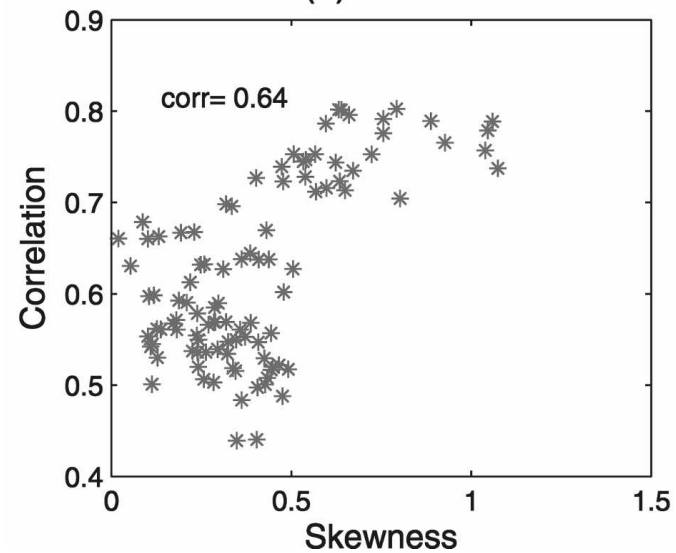

(b) ZC

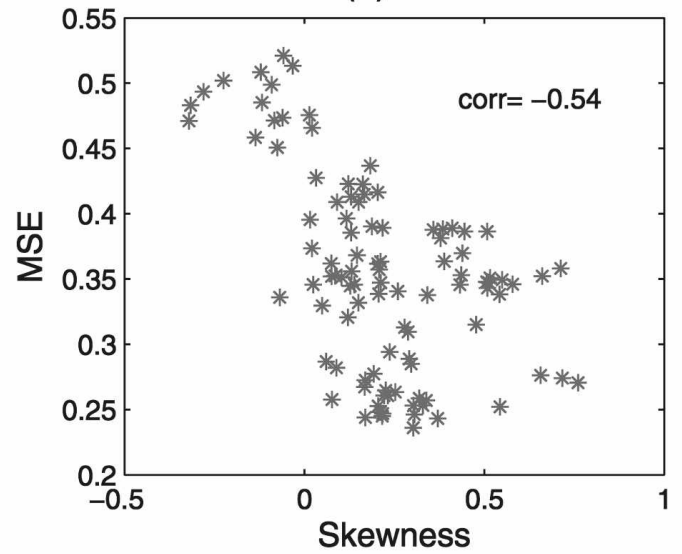

(d) HCM1

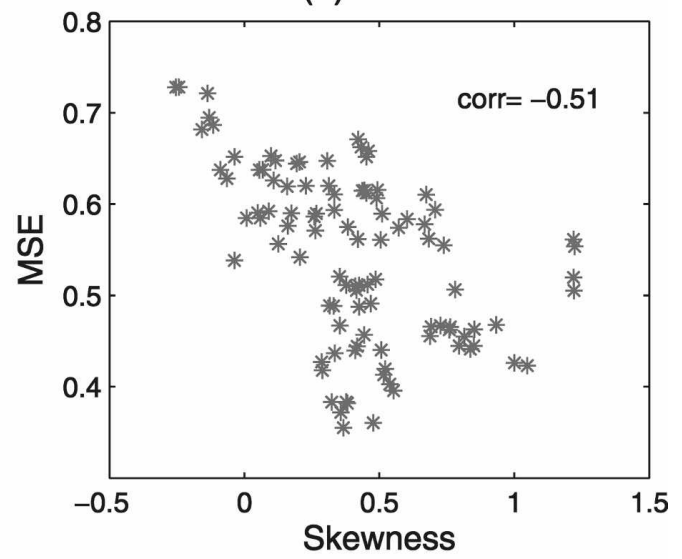

(f) $\mathrm{HCM} 2$

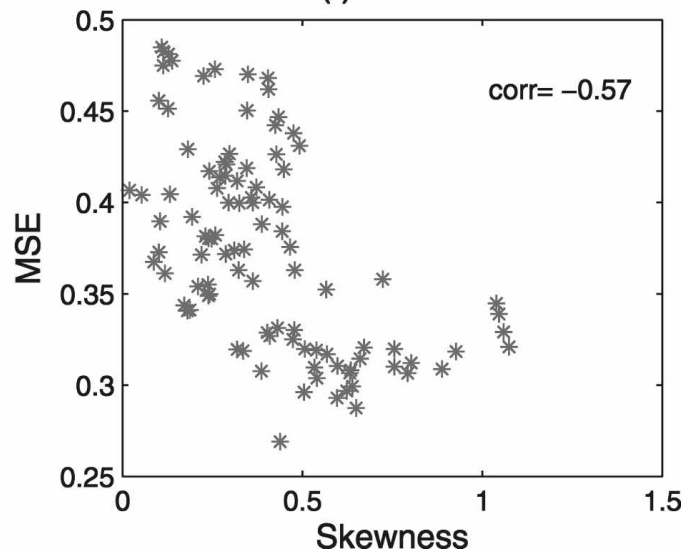

FIG. 12. Scatterplot of averaged prediction skill (left) $R$ and (right) MSE over lead times of 1-12 months against the skewness, both computed in each running window of $20 \mathrm{yr}$ from 1881 to 2000. The skewness was calculated using the analyzed Niño-3.4 SSTA index.

circulation models. In other words, the above nonlinearity analysis mainly addresses the asymmetric feature of ENSO variability, which is highly related to the strength of ENSO signals.

\section{c. Noise}

The noise usually means a high-frequency component with the lack of autocorrelation. While the "high 
ZC

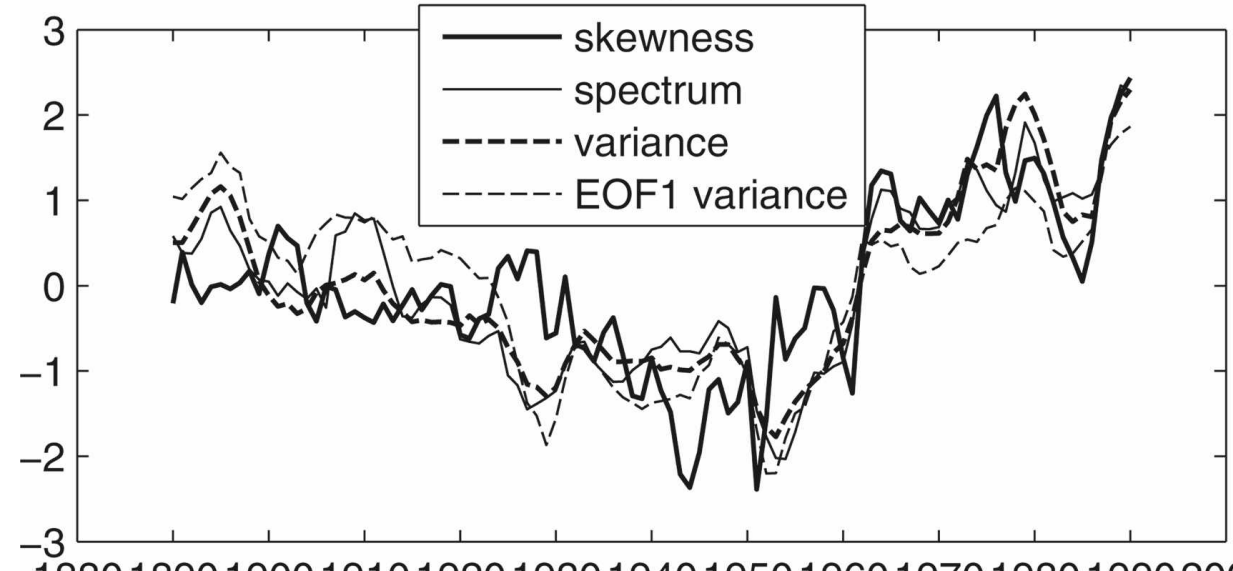

1880189019001910192019301940195019601970198019902000

HCM1
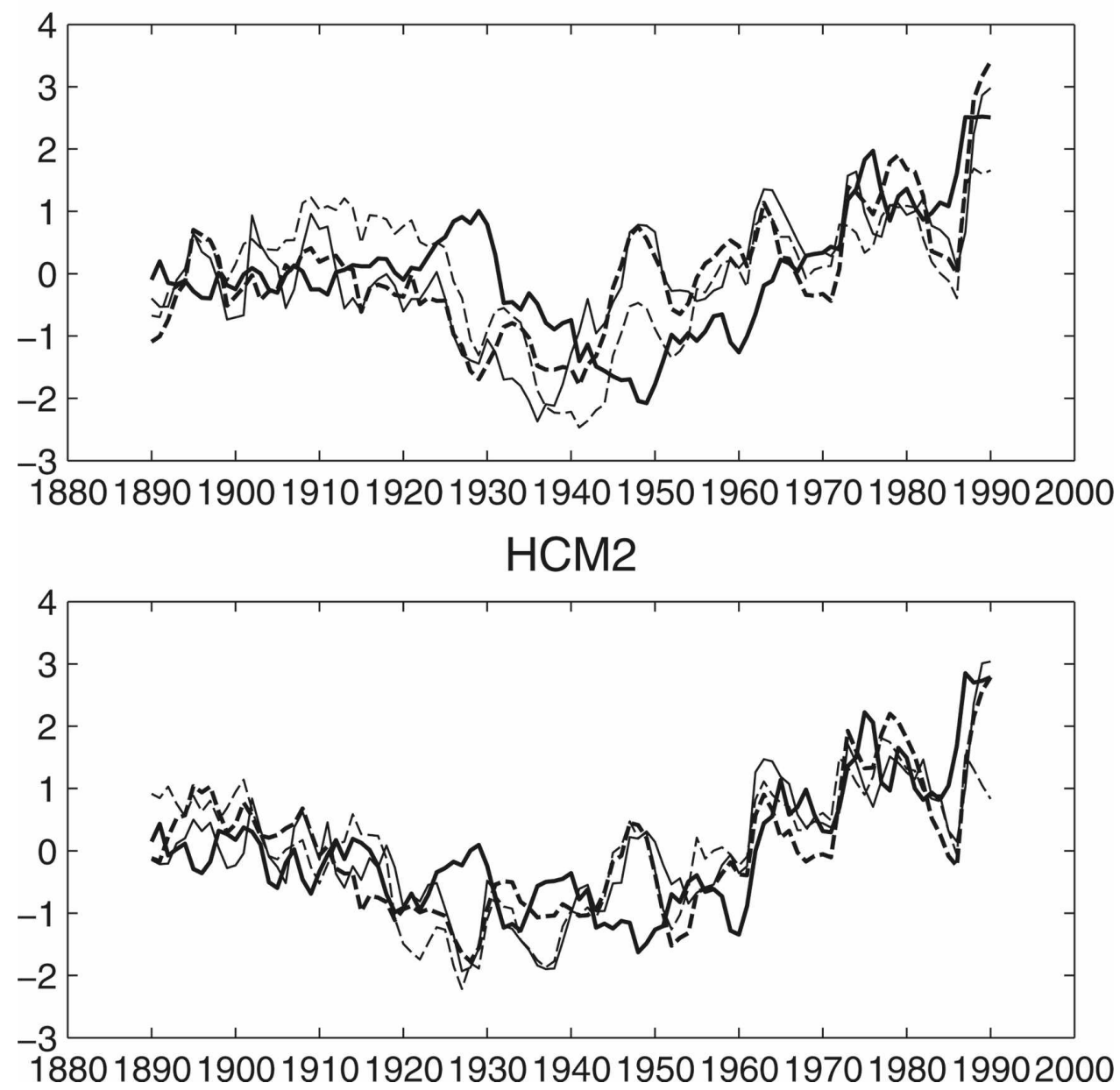

FIG. 13. Skewness, the power spectrum of ENSO frequencies at $2-5$-yr periods, and the variance explained by EOF1, calculated using the analyzed Niño-3.4 SSTA index and the tropical Pacific SSTA analysis in each running window of $20 \mathrm{yr}$ from 1881 to 2000. 
frequency" has a varied definition in different applications, it is often specified by a cutoff of periods of less than 3-12 months in the study of climate predictability (e.g., Kleeman and Moore 1997). Its strength can be measured by the variance of the high-frequency component. The noise is a primary source that limits atmospheric predictability. One might expect that the period that has a strong noise level should have a low atmospheric predictability, and vice versa. However, the noise may have a potential positive contribution to ENSO predictability because ENSO could be thought of as a linear, damped, stochastically forced system, as argued in many studies (e.g., Kleeman and Moore 1997; Penland and Sardeshmukh 1995; Chang et al. 1996; Moore and Kleeman 1999; Flügel et al. 2004; Tang et al. 2005). Some recent papers also mentioned the possible impact of MJO-WWB on ENSO (e.g., Zavala-Garay et al. 2005; Hendon et al 2007; Tang and Yu 2008). In this subsection, we will examine the relationship between the noise and ENSO predictability. It should be noted that the three models used here do not have internal noise; thus the observed noise impacts forecasts only through the initialization of predictions (called the initial impact hereafter). This "initial impact" could be further enhanced or weakened by internal dynamics of individual models. The noise-free nature of these models can allow us to identify the initial impact, but prevents a comprehensive study on the noise and ENSO predictability.

We first explore the initial impact by examining the noise strength of the initial SST. Figure 14 shows the variance of 3-month high-pass-filtered components of the analyzed Niño-3.4 SSTA index. Unlike signal and skewness, the interdecadal variation of noise is quite different between the $\mathrm{Z}-\mathrm{C}$ model and the other two models. As mentioned above, these models are noise free, and thus the initial noise characterized in Fig. 14 is mainly due to the different response of these models to the observed noise.

As can be seen in Fig. 15, the relationship of initial noise to predictability is model dependent and skill measure dependent. The noise generally has a positive relationship to $M S E$ skill, that is, large noise leading to poor skill (large MSE) and vice versa. However, the correlation coefficient between $R$ and the noise is small and is not statistically significant in all three models, as shown in the upper corner of each panel of Fig. 15.

The above initial impact only addresses how the initial noise impacts the predictability. Obviously, the noise involves not only the initialization state but also the developing stage of ENSO. To further explore possible impact of noise on the predictability, we repeat-

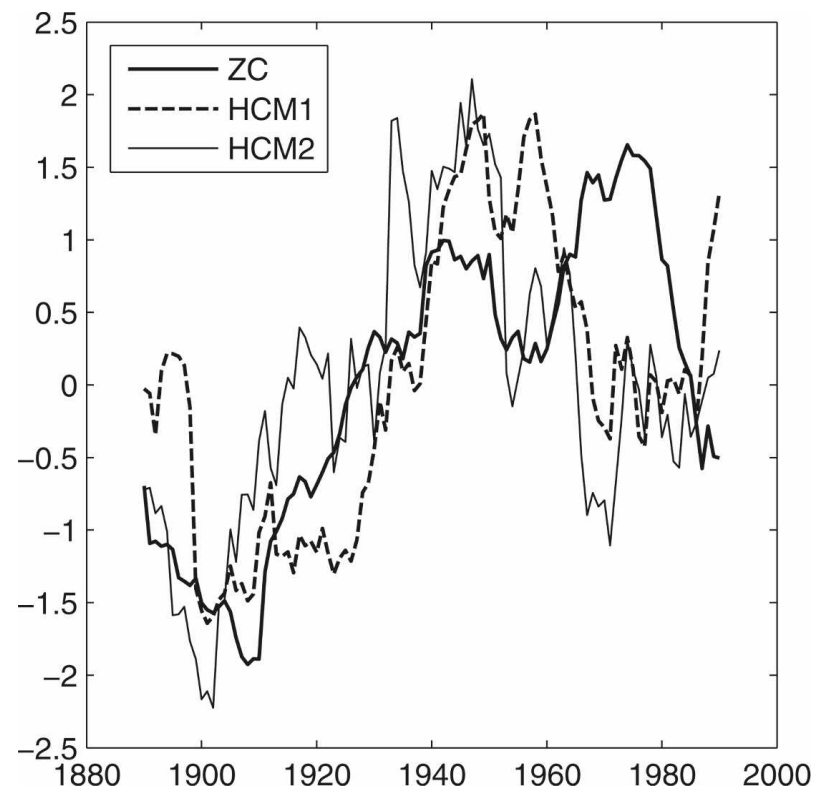

FIG. 14. The variance of noise of analyzed Niño-3.4 SSTA index of each model, calculated in each running window of $20 \mathrm{yr}$ from 1881 to 2000 . The noise data were obtained by a 3-month highpass filter.

edly run retrospective ENSO predictions for 1881-2000 using the three models, but added atmospheric noise to the individual noise-free atmosphere model (referred to as noisy atmosphere) during the whole period of each prediction. The extraction of the noise component from the time series is fraught with difficulties. Many different approaches have been devised in the literature (e.g., Priestley 1981). In this study, we restrict our attention to the wind stress component, as in Kleeman and Moore (1997). A noise dataset is thus obtained by applying a 3-month high-pass filter to the NCEP daily wind dataset from 1948 to 2000 . The stochastic component that is randomly taken each day from the noise dataset is added into individual atmospheric models to be coupled with the ocean model. Shown in Fig. 16 is the running skill from these retrospective predictions. As can be shown, the noisy atmosphere degrades MSE skill but affects correlation skill little, compared with the skill by the noise-free atmosphere. An interesting finding is that the noisy atmosphere does not change the feature of interdecadal variation in predictability, suggesting that the interdecadal variation in ENSO predictability is most probably due to the variation of ENSO signals, as discussed above.

Figure 16 was obtained by a 20 -yr running window, thereby representing an overall impact of noise on predictability during a 20 -yr period. This does not mean that stochastic noise always degrades prediction skill. 
(a) ZC

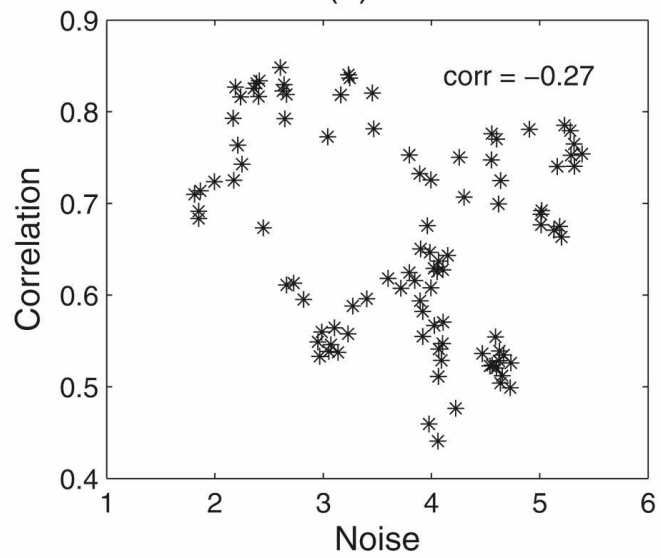

(c) HCM1

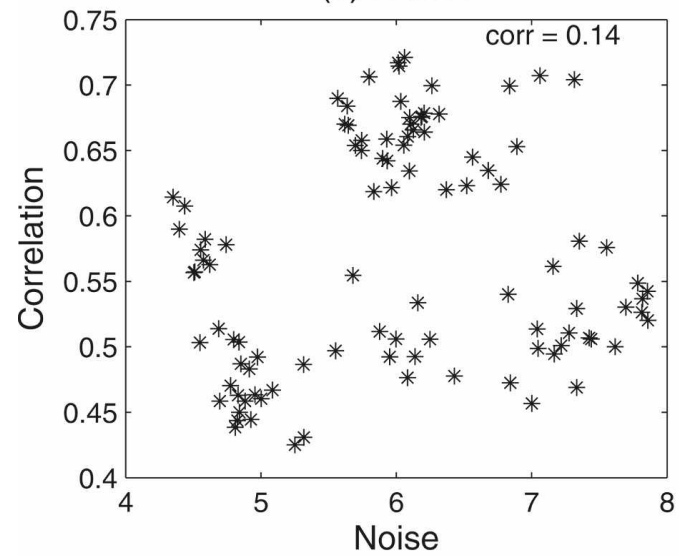

(e) $\mathrm{HCM} 2$

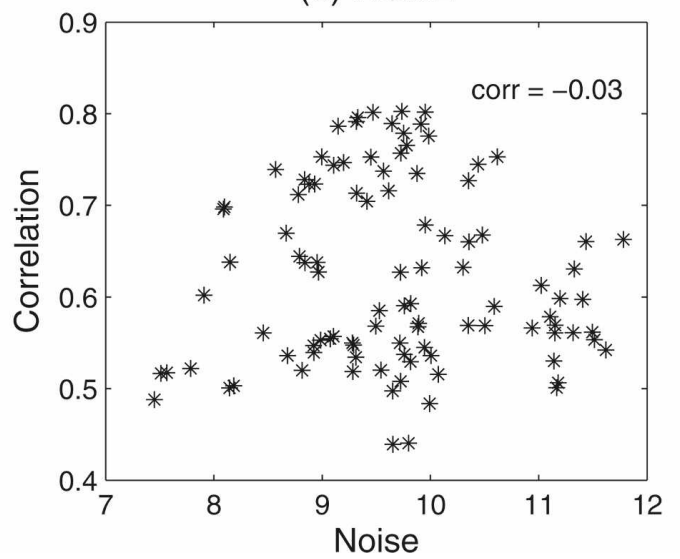

(b) ZC

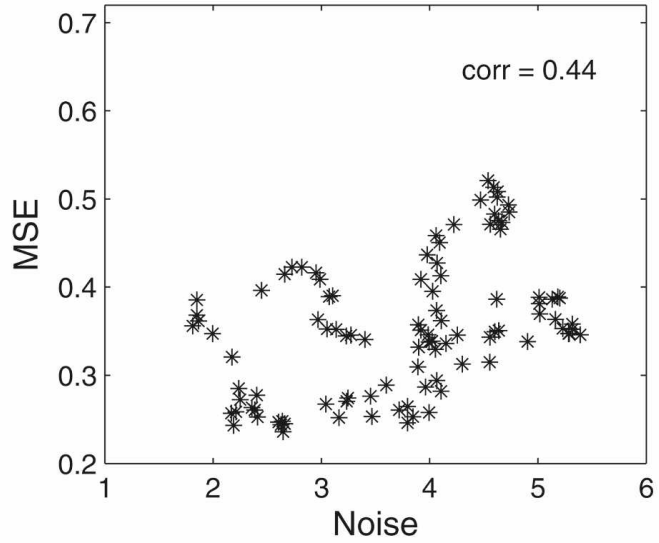

(d) HCM1

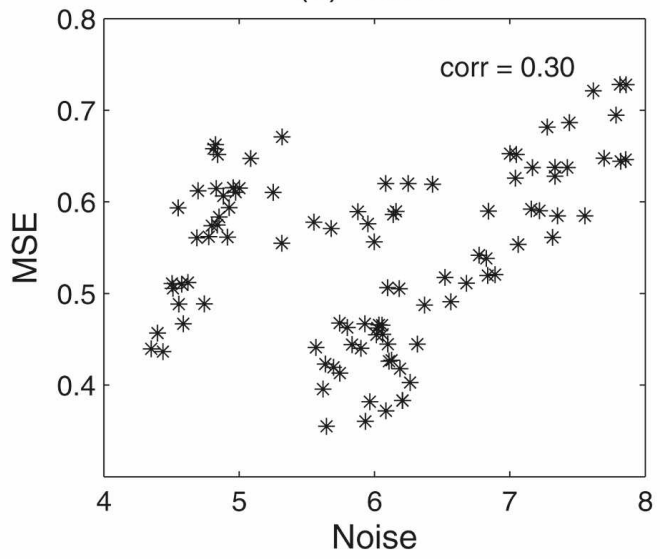

(f) $\mathrm{HCM} 2$

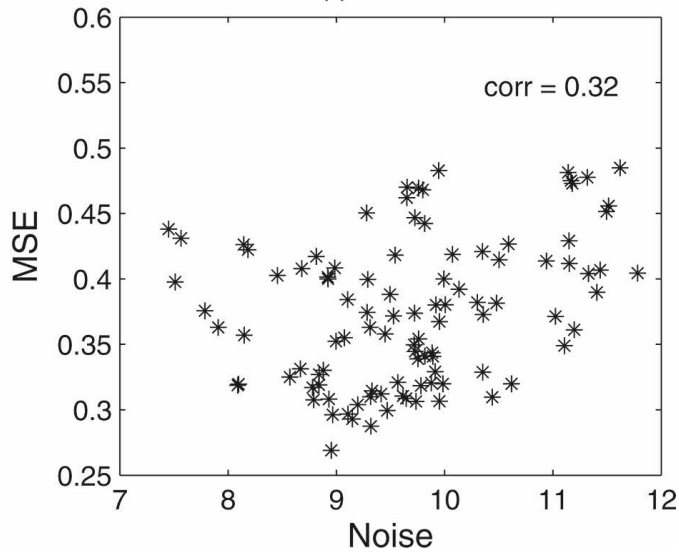

FIG. 15. Same as Fig. 10, but the skill against the variance of noise, which was obtained through 3-month high-pass filter.

We calculated the MSEIP for all of the prediction cases. Figure 17 shows the difference between MSEIP with a noisy atmosphere and MSEIP with a noise-free atmosphere, thereby a negative value indicates that the noisy atmosphere actually produces a better prediction. As can be seen, the noisy atmosphere is indeed able to lead to better predictions for the three models. In detail, the noisy atmosphere results in $39 \%, 30 \%$, and $35 \%$ of the 


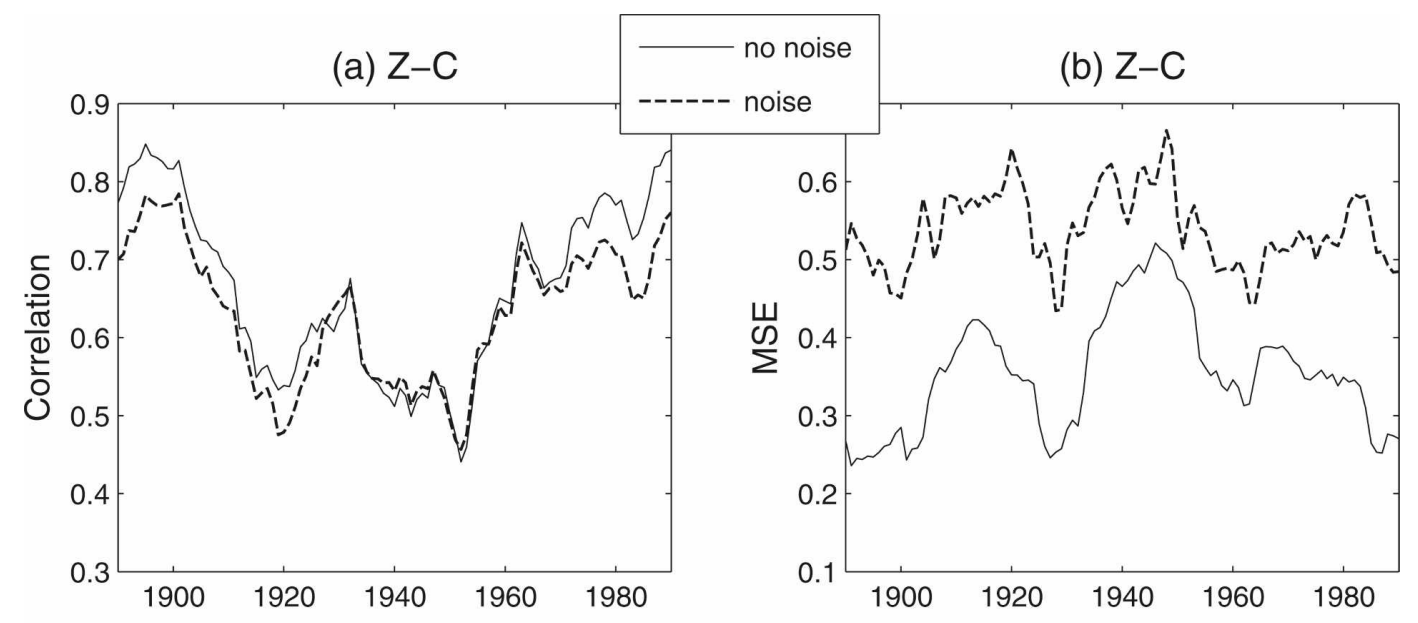

(c) $\mathrm{HCM} 1$

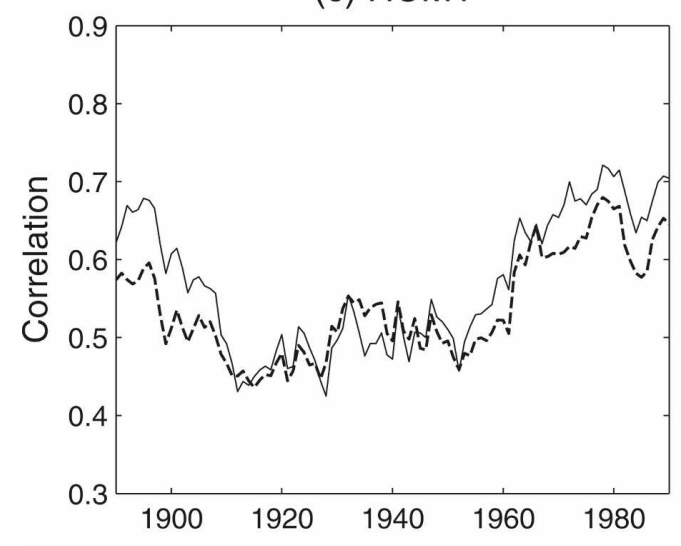

(e) HCM2

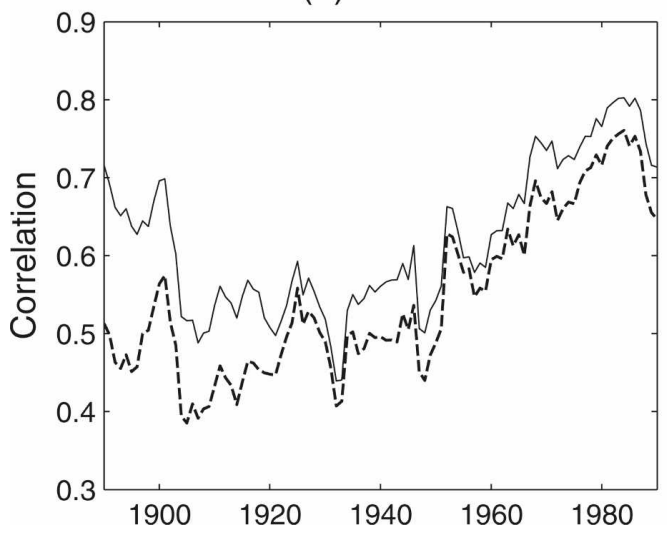

(d) HCM1

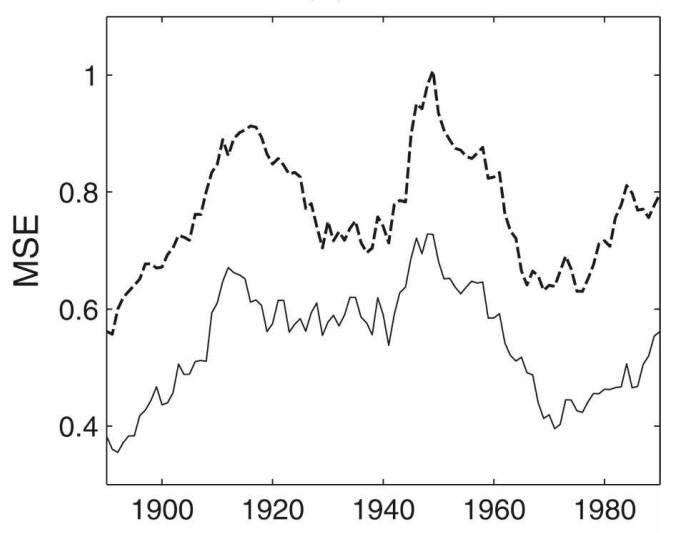

(e) $\mathrm{HCM} 2$

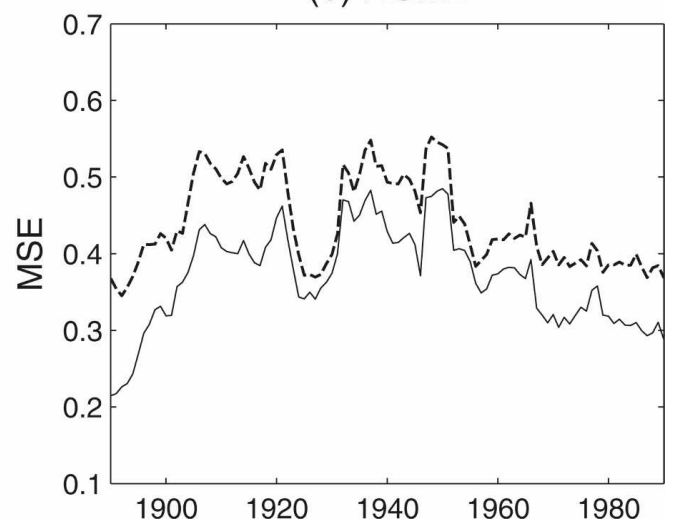

FIG. 16. Solid line is the same as Fig. 6 and dashed line is the skill of prediction where the atmospheric noise (white) was randomly superimposed into the wind stress during the prediction period.

total predictions having smaller MSEIP than the noisefree atmosphere for the $\mathrm{Z}-\mathrm{C}$ model, HCM1, and HCM2, respectively. Thus, the impact of noise on prediction skill could have two sides. On one hand, inclu- sion of noise might favor ENSO simulation and forecast, because the stochastic forcing can act to trigger and/or amplify ENSO oscillation as demonstrated by much theoretical and observational work (e.g., Moore 

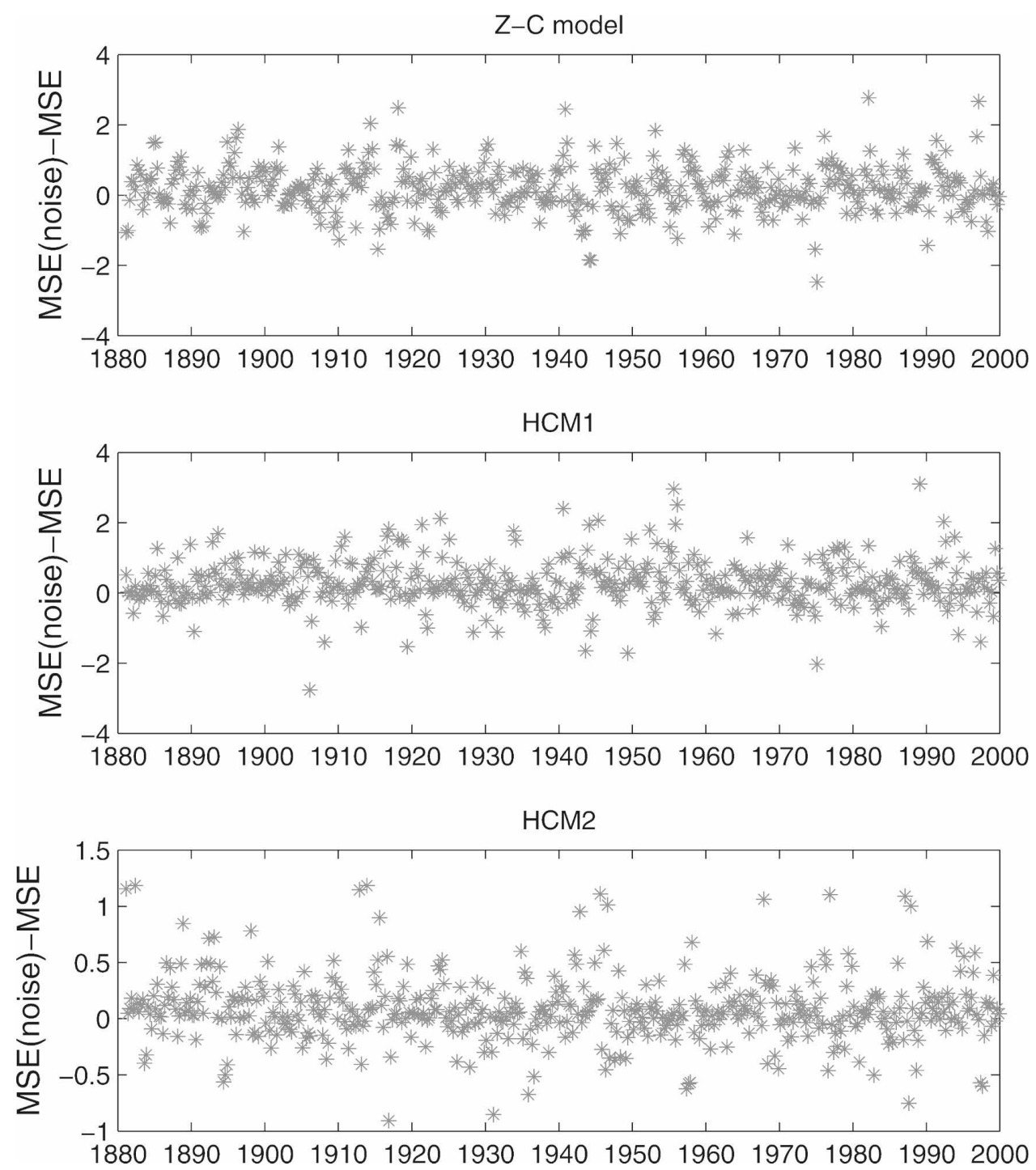

FIG. 17. MSEIP difference between two predictions, one with noisy atmosphere and the other with noise-free atmosphere, for (top) Z-C, (middle) HCM1, and (bottom) HCM2, as a function of initial time from 1881 to 2000 . A negative value indicates that the MSEIP with noisy atmosphere is smaller than that with noise-free atmosphere, and vice versa.

and Kleeman 1999; Flügel et al. 2004; Tang et al. 2005). On the other hand, however, the noise can dissipate the signals present in the initial condition, leading to the loss of predictability. Thus, the overall impact of noise might be determined by the both sides, and is probably model dependent. Also different types of stochastic noise have different impacts on dynamical systems like ENSO. Theory suggests that while additive stochastic forcing has a predetermined distribution, multiplicative forcing can alter dynamical characteristics and properties of the system (Perez et al. 2005). Perez et al. (2005) found, using an intermediate coupled model of ENSO, that multiplicative forcing pro- duces larger positive anomalies (El Niño) whereas additive forcing produces larger negative anomalies (La Niña).

We also performed another experiment, that is, considering the noise to be red. In this experiment, the atmospheric noise added into the atmospheric model consists of the noise of the current time step and that of the previous step. The results show that the prediction skill, both correlation and MSE, dramatically decrease as the noise is assumed to be red (not shown). This also indicates that the results shown in Figs. 16 and 17 might be sensitive to the definition of the noise and the method used to extract it. 


\section{Discussion and summary}

An important task of ENSO predictability studies is to derive robust conclusions based on multiple models and long-term retrospective forecasts that cover sufficient ENSO cycles. In this paper, long-term retrospective forecasts of the past $120 \mathrm{yr}$ were performed with three realistic ENSO models. With the assimilation of the historic SST dataset, all models display useful prediction skills.

An interesting finding from these retrospective forecasts is that all models show a consistent interdecadal variation in prediction skill. The prediction skill was high in the late nineteenth century from 1881 to 1900 , and then declined with time, reaching a minimum around the 1940s-50s, beyond which it rebounded and increased with time until the 1960s. All models had relatively high prediction skill from the 1960s, especially in the late twentieth century from 1981 to 2000. The similar results in these different models suggest that the interdecadal variability of ENSO predictability may exist generally.

Further analyses indicate that the interdecadal variation in predictability is highly related to the interdecadal variation of ENSO variability itself. In the late nineteenth century, ENSO variability was strong and all models showed high correlation skill and low MSE. Since then ENSO variability had weakened until the 1960s, during which all models showed the poorest prediction skill coincidentally. The middle-late twentieth century has the best prediction skill in all models, accompanying the strongest ENSO variability during this period. Such a good relationship between the strength of ENSO variability and its predictability is due to the critical impact of signals present in the initial conditions. When the initial signals are larger, they will be damped or dissipated more slowly, leading to relatively good predictions. Mathematically, because signals are large, more information will be produced compared with the climatological forecast, leading to a forecast that is likely to be reliable.

The nonlinearity and stochastic noise were generally thought to be the two most possible factors to limit ENSO predictability. Using the skewness as a measure of asymmetry that is able to characterize the nonlinearity, we found a good relationship between the asymmetry and prediction skill for the three models, that is, stronger asymmetry leads to better predictions. This is because the asymmetry is highly related to the strength of ENSO signals. The large asymmetry often results from strong ENSO events, leading to good predictions. An (2004) also pointed out that the interdecadal change in predictability was related to the interdecadal change in ENSO asymmetry. An and Jin (2004) showed that the nonlinear terms in the heat equation were responsible for the remarkable asymmetry between the warm and cold ENSO states. On the other hand, enhanced nonlinearity may be able to intensify the ENSO amplitude, leading to better prediction. However, because of the lack of a full spectrum of ENSO coupled models in this study, the nonlinearity analysis mainly addresses the asymmetric feature of ENSO variability, which is highly related to the strength of ENSO signals.

In contrast to the significant relationship between ENSO signal, the asymmetry, and the predictability, the impact of observed noise at initial time on the predictability is not very obvious, and is somehow model and measure dependent. This is probably because different models have varied responses to the initial noise resulting from high sensitivity of nonlinear models to external high-frequency forcing. For all three models, the noise at initial time has a positive relationship to MSE skill, that is, large noise leads to poor skill (large MSE). To explore possible impact of stochastic noise involving in the developing stage of ENSO, we run retrospective predictions with stochastic noise superimposed into the atmosphere models during the period of prediction. The results show that the stochastic noise degrades the overall skill, especially for MSE skill. However, stochastic noise can lead to better skill for some individual predictions. Thus, stochastic noise can impact predictability in two sides as mentioned before. On one hand, the stochastic noise could favor ENSO simulation and forecast because it can act to trigger and/or amplify ENSO oscillation, as evidenced in some recent papers on MJO/WWB and ENSO (e.g., ZavalaGaray et al. 2005; Gebbie et al. 2007; Tang and Yu 2008). On the other hand, the noise can dissipate the signals present in the initial condition, leading to the loss of predictability. Therefore, the stochastic forcing is probably an uncertain source to impact ENSO predictability.

Several cautions should be borne in mind. First, all models used in this study are either intermediate or hybrid models, and lack some necessary physical and dynamical processes that must be represented by more complicated models, such as coupled general circulation models (CGCMs). Thus, a whole spectrum of ENSO predictability needs further study using more complicated models. It is interesting to explore whether the results and findings presented in this study also exist in complicated GCM models. Second, the skewness discussed in this study mainly represents the nonlinear components characterized by asymmetric features of ENSO, which is highly related to the anomalies of ENSO variability. There are nonlinear components 
that may be important to ENSO but were not considered in our models. In particular, the nonlinearity was diagnosed only by oceanic analyses and observations in this study. Some important atmospheric nonlinearities were not taken into account such as MJO-ENSO interaction and the impact of WWB on ENSO. Third, we used a running window of $20 \mathrm{yr}$ to analyze interdecadal variations in predictability and other variables. The window length of $20 \mathrm{yr}$ was motivated by Chen et al. (2004), where the interdecadal variations in predictability were discussed in such an interval. We also performed several sensitivity experiments, with the window length of 10,30 , and $40 \mathrm{yr}$. The relationships of predictability to skewness, signal, and noise are similar to those presented in this paper. Finally, the stochastic noise used in the sensitivity experiment might not be able to completely represent realistic atmospheric stochastic forcing. The complicated CGCMs that can simulate realistic atmospheric transitions and weatherscale variability might be required to further study the role of stochastic forcing in ENSO predictability. Also, we used the 3-month high-pass filter to obtain noise in this study. One may argue that 3 months might not be an objective choice. These concerns need to be addressed through more comprehensive analyses. Nevertheless, this study is to date the first work to discuss ENSO predictability using multiple models and longterm predictions. The consistent results and conclusions found in the three models offer valuable insight to some important issues of ENSO predictability.

Acknowledgments. This work is supported by the Canadian Foundation for Climate and Atmospheric Sciences (CFCAS) through Grant GR-523 and the Canada Research Chair program.

\section{REFERENCES}

An, S. I., 2004: Interdecadal changes in the El Nino-La Nina asymmetry. Geophys. Res. Lett., 31, L23210, doi:10.1029/ 2004GL021699.

_ , and F.-F. Jin, 2004: Nonlinearity and asymmetry of ENSO. J. Climate, 17, 2399-2412.

Chang, P., L. Ji, H. Li, and M. Flügel, 1996: Chaotic dynamics versus stochastic process in El Nino-Southern Oscillation in coupled ocean-atmosphere models. Physica D, 98, 301-320.

Chen, D., M. A. Cane, A. Kaplan, S. E. Zebiak, and D. Huang, 2004: Predictability of El Nino in the past 148 years. Nature, 428, 733-736.

Deng, Z., and Y. Tang, 2008: Long-term ENSO hindcast in a hybrid coupled model (II): Variation of predictability. Climate Dyn., doi:10.1007/s00382-008-0398-2.

, - - and X. Zhou, 2008: Long-term ENSO hindcast in a hybrid coupled model (I): SST assimilation with ensemble Kalman filter. Climate Dyn., doi:10.1007/s00382-008-0399-1.
Eccles, F., and E. Tziperman, 2004: Nonlinear effects on ENSO's period. J. Atmos. Sci., 61, 474-482.

Flügel, M., P. Chang, and M. C. Penlend, 2004: The role of stochastic forcing in modulating ENSO predictability. $\mathrm{J}$. Climate, 17, 3125-3140.

Galanti, E., E. Tziperman, M. Harrison, A. Rosati, and Z. Sirkes, 2003: A study of ENSO prediction using a hybrid coupled model and the adjoint method for data assimilation. Mon. Wea. Rev., 131, 2748-2764.

Gebbie, G., I. Eisenman, A. Wittenberg, and E. Tziperman, 2007: Modulation of westerly wind bursts by sea surface temperature: A semistochastic feedback for ENSO. J. Atmos. Sci., 64, 3281-3295.

Goldenberg, S. D., and J. J. O'Brien, 1981: Time and space variability of tropical Pacific wind stress. Mon Wea. Rev., 109, 1190-1207.

Goswami, B. N., and J. Shukla, 1991: Predictability of a coupled ocean-atmosphere model. J. Climate, 4, 3-22.

Harrison, M. J., A. Rosati, B. J. Soden, E. Galanti, and E. Tziperman, 2002: An evaluation of air-sea flux products for ENSO simulation and prediction. Mon. Wea. Rev., 130, 723-732.

Hendon, H. H., M. Wheeler, and C. Zhang, 2007: Seasonal dependence of the MJO-ENSO relationship. J. Climate, 20, 531-543.

Horling, M. P., A. Kumar, and M. Zhong, 1997: El Niño, La Niña, and the nonlinearity of their teleconnections. J. Climate, 10, 1769-1786.

Jin, F.-F., J. D. Neelin, and M. Ghil, 1994: El Niño on the devil's staircase: Annual subharmonic steps to chaos. Science, 264, 70-72.

_, S.-I. An, A. Timmermann, and J. Zhao, 2003: Strong El Niño events and nonlinear dynamical heating. Geophys. Res. Lett., 30, 1120, doi:10.1029/2002GL016356.

Kirtman, B. P., and P. S. Schopf, 1998: Decadal variability in ENSO predictability and prediction. J. Climate, 11, 28042822.

Kleeman, R., 2002: Measuring dynamical prediction utility using relative entropy. J. Atmos. Sci., 59, 2057-2072.

- and A. M. Moore, 1997: A theory for the limitation of ENSO predictability due to stochastic atmospheric transients. J. Atmos. Sci., 54, 753-767.

Latif, M., J. Biercamp, H. von Storch, M. J. McPhaden, and E. Kirk, 1990: Simulation of ENSO-related surface wind anomalies with an atmospheric GCM forced by observed SST. $J$. Climate, 3, 509-521.

—_, and Coauthors, 1998: A review of the predictability and prediction of ENSO. J. Geophys. Res., 103, 14 375-14 393.

Madec, G., P. Delecluse, M. Imbard, and C. Levy, 1998: OPA 8.1 Ocean General circulation model reference manual. Institut Pierre Simon Laplace (IPSL), $91 \mathrm{pp}$.

Monahan, A. H., and A. Dai, 2004: The spatial and temporal structure of ENSO nonlinearity. J. Climate, 17, 3026-3036.

Moore, A. M., and R. Kleeman, 1999: Stochastic forcing of ENSO by the intraseasonal oscillation. J. Climate, 12, 1199-1220. , and Coauthors, 2006: Optimal forcing patterns for coupled models of ENSO. J. Climate, 19, 4683-4699.

Peng, P., and A. Kumar, 2005: A large ensemble analysis of the influence of tropical SSTs on seasonal atmospheric variability. J. Climate, 15, 1068-1085.

Penland, C., and P. D. Sardeshmukh, 1995: The optimal growth of tropical sea surface temperature anomalies. J. Climate, 8 , 1999-2024.

Perez, C. L., A. M. Moore, J. Zavala-Garay, and R. Kleeman, 
2005: A comparison of the influence of additive and multiplicative stochastic forcing on a coupled model of ENSO. $J$. Climate, 18, 5066-5085.

Priestley, M. B., 1981: Spectral Analysis and Time Series. Academic Press, 890 pp.

Rosati, A., K. Miyakoda, and R. Gudgel, 1997: The impact of ocean initial conditions on ENSO forecasting with a coupled model. Mon. Wea. Rev., 125, 754-772.

Smith, T. M., and R. W. Reynolds, 2004: Improved extended reconstruction of SST (1854-1997). J. Climate, 17, 2466-2477.

Syu, H.-H., and D. Neelin, 2000: ENSO in a hybrid coupled model. Part II: Prediction with piggyback data assimilation. Climate Dyn., 16, 35-48.

Tang, Y., 2002: Hybrid coupled models of the tropical PacificInterannual variability. Climate Dyn., 19, 331-342.

- , and W. W. Hsieh, 2002: Hybrid coupled models of the tropical Pacific-ENSO prediction. Climate Dyn., 19, 343-353.

— , and R. Kleeman, 2002: A new strategy for assimilating SST data for ENSO prediction. Geophys. Res. Lett., 29, 1841, doi:10.1029/2002GL014860.

—_, and W. W. Hsieh, 2003: ENSO simulation and predictions using a hybrid coupled model with data assimilation. J. Japan Meteor. Soc., 81, 1-19.

- and B. Yu, 2008: MJO and its relationship to ENSO. $J$. Geophys. Res., 113, D14106, doi:10.1029/2007JD009230.
_ W. W. Hsieh, B. Tang, and K. Haines, 2001: A neural network atmospheric model for hybrid coupled modeling. Climate Dyn., 17, 445-455.

_ , R. Kleeman, and A. Moore, 2004: SST assimilation experiments in a tropical Pacific Ocean model. J Phys. Oceangr., 34, 623-642.

,-- , and -2005 : On the reliability of ENSO dynamical predictions. J. Atmos. Sci., 62, 1770-1791.

-, H. Lin, and A. M. Moore, 2008: Measuring potential predictability in ensemble climate prediction. J. Geophys. Res., 113, D04108, doi:10.1029/2007JD008804.

Vecchi, G. A., and D. E. Harrison, 2003: On the termination of the 2002-03 El Niño event. Geophys. Res. Lett., 30, 1946, doi:10.1029/2003GL017564.

$\mathrm{Wu}$, A., and W. W. Hsieh, 2003: Nonlinear interdecadal changes of the El Niño-Southern Oscillation. Climate Dyn., 21, 719730 .

Zavala-Garay, J., C. Zhang, A. M. Moore, and R. Kleeman, 2005: The linear response of ENSO to the Madden-Julian oscillation. J. Climate, 18, 2441-2459.

Zheng, F., J. Zhu, and R.-H. Zhang, 2007: Impact of altimetry data on ENSO ensemble initializations and predictions. Geophys. Res. Lett., 34, L13611, doi:10.1029/2007GL030451. 
Copyright of Journal of Climate is the property of American Meteorological Society and its content may not be copied or emailed to multiple sites or posted to a listserv without the copyright holder's express written permission. However, users may print, download, or email articles for individual use. 